\title{
El tercer ciclo del carbón en Chile, de 1973 a 2013: del climaterio al rejuvenecimiento*
}

\section{The Third Cycle of Coal in Chile, from 1973 to 2013: From Climacteric to Rejuvenation}

\author{
César Yáñez y Martín Garrido-Lepe \\ Universidad de Valparaíso, Valparaíso, Chile, \\ emails: cesar.yanez@uv.cl; martin.garrido.lepe@gmail.com
}

\begin{abstract}
Resumen. A diferencia de otros latecomers, la historia energética chilena ha estado profundamente vinculada al consumo de carbón mineral. Su importancia en la matriz energética disminuiría desde 1953, abandonando industrias y ferrocarriles para concentrarse en la generación de gas y electricidad. Esta transición daría inicio a un nuevo ciclo de vida del mineral, caracterizado por un estrecho vínculo con la generación termoeléctrica. Desde 1990, el incremento de la demanda eléctrica dinamizó el consumo de carbón a niveles nunca antes vistos. El objetivo de este artículo es analizar el consumo de carbón mineral en la economía chilena durante los últimos 40 años. La hipótesis defendida es que, después de un declive en el consumo de carbón a fines de la década de 1960, se abre una transición caracterizada por el uso del mismo para la generación termoeléctrica.
\end{abstract}

Palabras clave: historia de la energía; energía; carbón; historia del carbón; historia económica de Chile.

Abstract. Unlike other latecomers, Chilean energy history has been deeply linked to the consumption of coal. Its importance in the energy matrix would decrease since 1953 , leaving behind industries and railroads to concentrate on the generation of gas and electricity. This transition initiated a new cycle of coal, characterized by a link with thermoelectric generation. Since 1990, the growth of electric demand has grown coal consumption to the highest levels in history. The objective of this article is to analyze the consumption of coal in the Chilean economy during the last forty years. The hypothesis is that after the decline of coal consumption in the late 1960s, a new era begins in which coal is used to generate thermoelectricity.

* Este artículo es el resultado de una investigación financiada por el proyecto ConiCYT núm. 1161425, Historia de las Transiciones Energéticas y el Cambio Estructural en la Economía Chilena (siglos XIX a XXI), en el que Yáñez es investigador responsable y Garrido-Lepe, ayudante de investigación. Una primera versión de este trabajo fue presentada en las VIJornadas Uruguayas de Historia Económica Reconstruir el Pasado. Entender el Futuro, desarrolladas entre el 2 y 4 de diciembre de 2015, en Montevideo, Uruguay. Agradecemos los comentarios realizados por Reto Bertoni, María del Mar Rubio, Joao Rodríguez Neto y Cecilia Zuleta. Todos los déficits que perduren en esta versión son de exclusiva responsabilidad de los autores.

Am. Lat. Hist. Econ., sep.-dic., 2017, pp. 224-258 | DOI: 10.18232/alhe.v24i3.833 
Key words: history of the energy; energy; coal; coal history; economic history of Chile.

JEL: N8; N9; N56; N96; Q4; Q48.

Fecha de recepción: 2 de noviembre de 2016. Fecha de aceptación: 26 de abril de 2017.

\section{INTRODUCCIÓN: LOS TRES CICLOS DEL CARBÓN:}

\section{EL CONSUMO EN EL LARGO PLAZO}

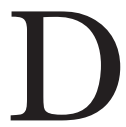
urante centenares de miles de años, la humanidad sólo pudo usar como fuente de energía la que provenía de las plantas, que a su vez dependían del Sol. Todos los conversores de energía disponibles eran orgánicos, transformando las plantas en calor, luz o movimiento. Entre todos, su propio cuerpo era la herramienta más importante disponible por las personas para transformar los alimentos en las diversas formas de energía (Walpole et al., 2012). Esto cambió cuando la máquina de vapor, alimentada con carbón fósil, puso en marcha la revolución industrial en Gran Bretaña durante la segunda mitad del siglo XVIII (Wrigley, 2013). Desde entonces, el carbón mineral ha acompañado los procesos económicos en una espiral de constantes innovaciones tecnológicas del que no hay señales que hagan pensar que se detendrá (Freeze, 2003; Kalder, Malanima y Warde, 2014; Smil, 2010).

Tuvieron que transcurrir unas décadas para que el consumo de carbón mineral penetrara en las economías latinoamericanas. Fueron las importaciones de combustible para la industria azucarera cubana y la minería chilena las que abrieron el camino (Yáñez, 2012). Una estimación detallada del consumo de energías modernas (carbón, petróleo, gas e hidroelectricidad) entre 1860 y 1925 para los países latinoamericanos puede verse en Rubio, Yáñez, Folchi y Carreras (2009). Y específicamente para el carbón, véase Yáñez, Rubio, Jofré y Carreras (2013). Ambos trabajos destacan el papel modernizador del carbón en las economías latinoamericanas. Mientras el trabajo de Yáñez y Carreras (2012) pone de relieve la diversidad de procesos en que el ferrocarril, la minería y la industria agroalimentaria transformaron unas economías tradicionales. En una tradición muy semejante se inscriben los estudios sobre consumo de energía en Uruguay, que incluyen el carbón mineral junto con las otras energías modernas, destacando la peculiaridad de la introducción de innovaciones técnicas en economías periféricas del sistema económico mundial (Bertoni, 2010; y Bertoni y Willebald, 2015; Rubio y Bertoni, 2008). Bertoni y Román (2013) abordaron el caso del carbón mineral en Uruguay, llegando a la conclusión que su importancia está focalizada en las primeras décadas del 
siglo XX como combustible para el transporte, la industria y la producción eléctrica, siendo sustituido por el petróleo a lo largo de las cuatro siguientes décadas. Como veremos más adelante, dicha aportación es una importante fuente de comparación con los ciclos observados en el caso chileno.

Este trabajo analizará el consumo de carbón en Chile desde 1973 en adelante, durante lo que llamamos el tercer ciclo del carbón, en el que el consumo de este combustible fósil alcanzó las cotas más altas de la historia, acentuó su dependencia externa y dejó de ser de consumo final ${ }^{1}$ para dedicarse mayoritariamente a la producción de energía secundaria (electricidad). La relevancia de estudiar el carbón mineral en esta época reside, en primer lugar, a su importancia dentro de la matriz energética de Chile. Importancia que es cuantitativa, llegando a representar más de $50 \%$ del total de las energías primarias utilizadas en la generación de electricidad y más de 20\% del total de energías primarias consumidas en Chile. La construcción de centrales térmicas a carbón estuvo estrechamente ligada a la demanda de energía eléctrica en zonas donde era inviable la construcción de centrales hidroeléctricas, y en momentos en que el incremento del petróleo y sus derivados era cada vez más costoso, después de 1973. Sin el carbón, la generación eléctrica chilena no habría seguido la senda descrita. Esto conecta con los aspectos cualitativos. La economía chilena sufrió restricciones eléctricas por carecer de inversiones en centrales más modernas que las carboneras y quedó amarrada a una tecnología que entraba en obsolescencia a pasos agigantados. Más aún, en esta época las minas chilenas de carbón de las regiones tradicionales (Bio-Bio y Arauco) agotaron sus reservas y el país se vio obligado a importar carbón, hecho subsanado en parte por la producción de la mina Invierno en la región de Magallanes. Sin embargo, esto sólo ha atenuado la grave dependencia energética, sin llegar a eliminarla. Es imprescindible señalar que este ciclo aún está en desarrollo y que es difícil aventurarse a indicar cuál será su final.

Las últimas investigaciones que se han publicado sobre la historia energética chilena nos han permitido, entre otras cosas, reconstruir la trayectoria del carbón en la economía chilena desde 1844 hasta $1960 .^{2}$ Este exten-

\footnotetext{
${ }^{1}$ Se entiende por consumo final toda aquella energía utilizada directamente en la generación de trabajo, sin sufrir más transformaciones antes de ser empleada.

${ }^{2}$ Los estudios que han abordado la trayectoria energética chilena pueden agruparse en dos categorías: aquellas que la tratan en el contexto latinoamericano, y las que se han especializado exclusivamente en el caso chileno. En el primer grupo, es necesario mencionar los aportes de Folchi y Rubio (2006), Jofré (2012), Rubio, Yáñez, Folchi y Carreras (2009), Rubio y Folchi (2012), Rubio y Tafunell (2014), y Yáñez, Rubio, Jofré y Carreras (2013). En cuanto al segundo grupo, se encuentran los trabajos de Garrido (2015), Yáñez y Garrido (2015) y Yáñez y Jofré (2011). Los trabajos de Jofré (2012), Yáñez, Rubio, Jofré y Carreras (2013), Yáñez y Garrido (2015) y Yáñez y Jofré (2011), ofrecen una base estadística que nos permite reconstruir la trayectoria de la historia del carbón, tanto del consumo, como la producción, exportaciones e importaciones entre 1844 y
} 
so periodo abarca desde los primeros intentos de la economía chilena por insertarse en la era de la cinética y adoptar las tecnologías derivadas de la revolución industrial, pasando por los cambios y efectos sufridos durante el periodo de entreguerras hasta el primer periodo de la industrialización dirigida por el Estado. La presente investigación continúa con el estudio de esta trayectoria, incorporando el análisis de los datos que se extienden entre 1960 y 2013, con el fin de comprender los cambios y comportamientos de la producción, consumo y distribución del carbón mineral entre los diversos sectores de la economía chilena. El análisis de los datos agregados permite visualizar la existencia de tres ciclos del consumo de carbón mineral a lo largo de estos casi 170 años de historia. ${ }^{3}$ La trayectoria del consumo del carbón mineral en Chile entre 1844 y 2013 está caracterizada por la periodificación de cada uno de los tres ciclos identificados (véase gráfica 1). Estos se desarrollan entre 1844 y 1929 el primero, 1930 y 1973 el segundo, y 1973 hasta la actualidad el tercero. ${ }^{4}$

El primero de los ciclos del carbón ha sido analizado por Yáñez y Jofré (2011) y Yáñez et al. (2013). Allí, los autores identifican los primeros intentos de la economía chilena por insertarse en la era de la cinética moderna. El proceso chileno se ubicaría dentro de los primeros en el panorama latinoamericano, situación que se habría facilitado por la explotación del mineral en la zona centro y sur de Chile, que se sumaría a la importación de carbones principalmente británicos y australianos. Durante este primer ciclo, el consumo de carbón mineral registró un máximo histórico de 1775000 toneladas equivalentes de petróleo (TEP $)^{5}$ en 1912, que sólo sería superado en 1988. Sin embargo, sería durante la primera guerra mundial

1960 en Chile. El trabajo de Yáñez y Garrido (2015) permite conocer a fondo la distribución del consumo de carbón nacional entre 1933 y 1960.

${ }^{3}$ En el caso uruguayo estudiado por Bertoni y Román (2013) hay semejanzas y diferencias. Aunque ellos no focalizan su estudio de la misma forma que nosotros puesto que se centran en el estudio de la transición del carbón al petróleo, podría decirse que hay también tres ciclos que grosso modo coinciden en el tiempo con los observados en Chile, pero con comportamientos diferentes. Esto se explica por el hecho de que Chile, a diferencia de Uruguay, ha sido históricamente productor de carbón, mientras que Uruguay ha carecido del todo de este recurso natural. Este factor fue señalado antes en el trabajo comparativo de Uruguay y Nueva Zelanda de Bertoni y Willebald (2015).

${ }^{4}$ En la determinación de los ciclos históricos del consumo de carbón mineral por la economía chilena, hemos tenido en cuenta la evolución de sus tasas de crecimiento, el uso que se hace de este combustible y el origen nacional o extranjero del combustible (dependencia energética). A diferencia de Stevens (2000), que propone mirar las dos primeras variables, agregamos la dependencia energética por la importancia que tiene en la caracterización de los ciclos chilenos del carbón.

${ }^{5}$ La tonelada equivalente de petróleo (TEP) corresponde a la cantidad de energía liberada en la combustión de una tonelada de petróleo y, según la Agencia Internacional de Energía, equivale a 107 Kcal. En esta investigación, el factor de conversión entre la tonelada de carbón y petróleo corresponde a 0.7 . 
(1914-1918) cuando iniciaría el fin de este primer ciclo, producto del desarrollo de la transición energética de los combustibles fósiles. Este ciclo terminaría abruptamente con la crisis económica de 1929, dando inicio al segundo ciclo del carbón.

El mérito de esta etapa fue haber iniciado la modernización de la economía chilena, dejando atrás progresivamente los rasgos de una economía exclusivamente orgánica. Los buenos resultados de la economía chilena del siglo XIX (véanse Bértola, 2011; Bértola y Ocampo, 2010) deben considerar los cambios técnicos inducidos por el consumo de carbón mineral en el transporte, la minería y la industria (véase gráfica 1).

El segundo ciclo del carbón chileno ${ }^{6}$ se inició poco después de la Gran Depresión de 1929, y terminó en 1973, cuando la primera crisis del petróleo ocasionó diversos transtornos en la matriz energética chilena, específicamente en la generación de energía eléctrica. Las características de este segundo ciclo merecen especial atención pues son fundamentales para comprender los cambios experimentados durante el tercer ciclo (véase cuadro 1).

En resumen, durante el segundo ciclo, la principal fuente de abastecimiento del consumo de carbón mineral recayó casi exclusivamente en la producción nacional, reduciendo las importaciones a menos de 1\% del total consumido; el consumo del carbón mineral tendió a concentrarse en dos actividades -industria y transporte-, representando ambas más de $60 \%$ del consumo total de carbón hasta 1960; y finalmente, hasta 1960, más de $80 \%$ del carbón que Chile consumía era consumo final, cantidad que se reduce a $46 \%$ en 1970 .

La crisis de 1929 redujo las exportaciones chilenas y los precios de los productos exportados, disminuyendo considerablemente la cantidad de divisas existentes en el país. Las importaciones se tornaron cada vez más caras y difíciles de realizar, afectando fuertemente la provisión de bienes de consumo final y de materias primas. Esta situación fue el motivo central para redirigir el modelo de desarrollo hacia el mercado interno y hacia la industrialización (Bértola, 2011, p. 258). ${ }^{7}$ La reducción de las importacio-

${ }^{6}$ Un estudio más acabado del segundo ciclo de carbón mineral se encuentra en Yáñez y Garrido (2015). Dicha investigación plantea que, durante este segundo ciclo, aquellos sectores que consumieron carbón final una vez que el petróleo y la electricidad ofrecieran alternativas económicamente más eficientes, presenciaron algunas dificultades para realizar procesos de modernización energética, que podrían haberse traducido en mejoras de productividad. El periodo estudiado abarcó desde 1933 hasta 1960, pues la falta de datos no permitió extender aún más la investigación. Sin embargo, desde su presentación hasta la redacción del presente artículo, la incorporación de nuevos datos ha permitido visualizar mejor la trayectoria del carbón en los siglos XIX y XX, permitiendo comprender que el segundo ciclo del carbón podría extenderse hasta 1973, y no 1960 como se planteaba anteriormente. Durante estos años, ocurrió también la transición desde el carbón al petróleo, modificando la matriz energética chilena, haciéndola más compleja.

${ }^{7}$ Según Luis Bértola (2011, p. 264), el periodo comprendido entre 1929 y 1945 fue, para América Latina, un periodo de transición entre el modelo primario-exportador y el modelo de 


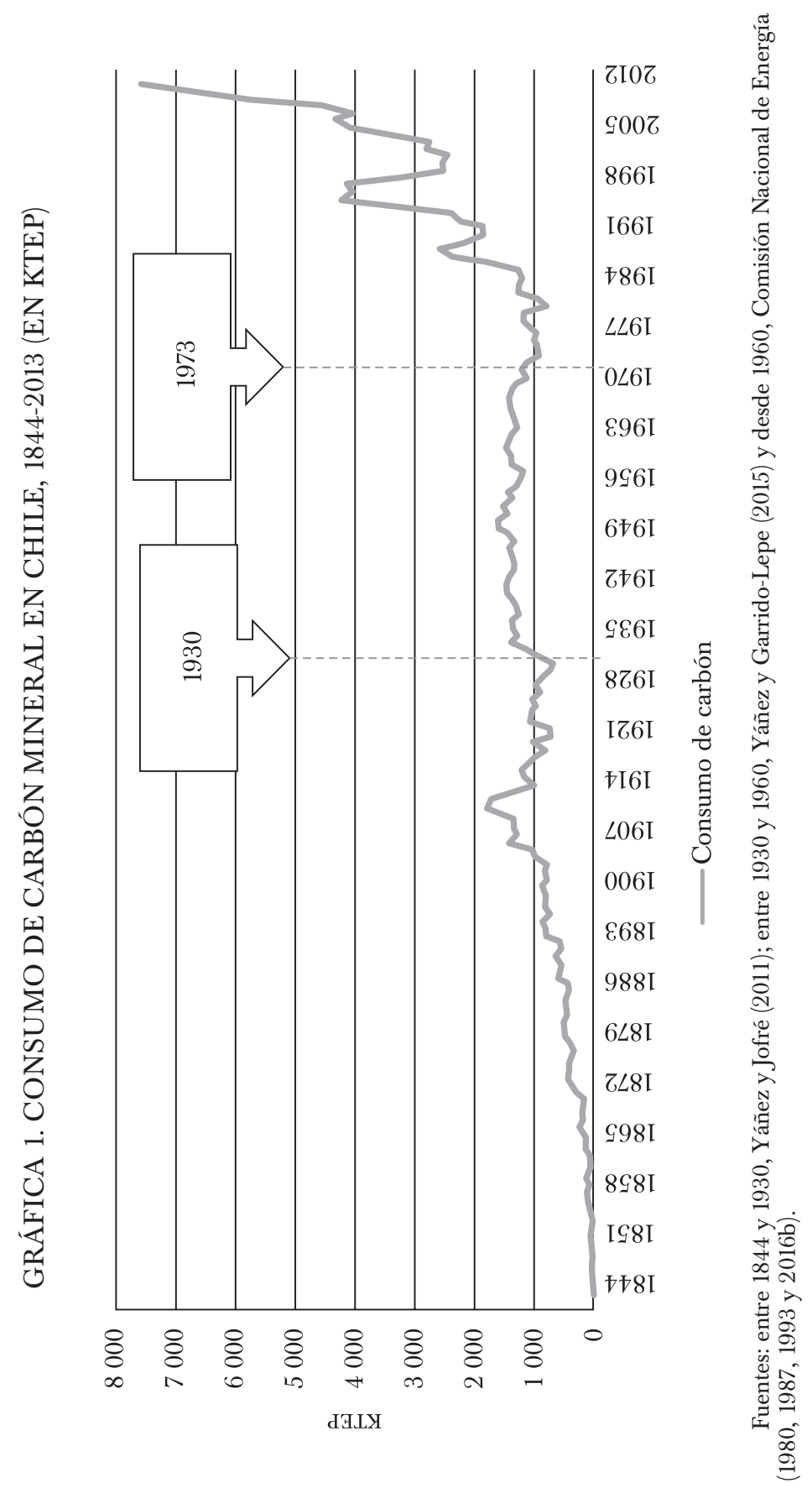




\section{CUADRO 1. CONSUMO, PRODUCCIÓN Y DISTRIBUCIÓN \\ DE CARBÓN MINERAL EN CHILE DURANTE EL SEGUNDO CICLO DE CARBÓN (EN KTEP)}

\begin{tabular}{lrrrrr}
\hline & 1933 & 1940 & 1950 & 1960 & 1970 \\
& & & & & \\
& 1031 & 1298 & 1485 & 986 & 1092 \\
Producción nacional & 926 & 1277 & 1433 & 991 & 1351 \\
Consumo total & 111 & 102 & 104 & 99 & 81 \\
$\quad$ Consumo total (porcentaje) & & & & & \\
& 450 & 449 & 444 & 291 & 173 \\
Transportes & 49 & 35 & 31 & 29 & 13 \\
$\quad$ Consumo total (porcentaje) & 233 & 507 & 673 & 464 & 475 \\
Industrias & 25 & 40 & 47 & 47 & 28 \\
$\quad$ Consumo total (porcentaje) & & & & & \\
& 74 & 75 & 78 & 76 & 41 \\
Consumo total (porcentaje) & & & & & \\
$\quad$ transportes+industria & 990 & 1072 & 1208 & 848 & 625 \\
Consumo final & 84 & 84.2 & 86 & 46 \\
$\quad$ Consumo total (porcentaje) & & & & & \\
\hline
\end{tabular}

Fuentes: entre 1933 y 1960, Dirección General de Estadísticas, Anuario Estadístico de la República de Chile (diversos años), sección Minería, y para 1970, Comisión Nacional de Energía (1980, 1987).

nes de petróleo fue rotunda, disminuyendo su consumo de 1100 KTEP en 1929, a 264 en 1933, recuperando sus niveles previos a la crisis en 1946. Muy diferente fue el caso del carbón. Desde 1918, casi 100\% del carbón mineral que Chile consumía fue abastecido por la producción nacional, razón por la cual el impacto de la crisis en las importaciones de este mineral fue menos alarmante. Así fue como, sin muchas posibilidades de importar energía y con unas minas de carbón ubicadas a poco más de 500 kilómetros de la capital, la economía nacional resolvería sus necesidades energéticas mediante el uso del carbón mineral chileno, lo que obligó a incrementar la producción de dichas minas hasta los máximos niveles de su historia. De esta forma, el abastecimiento del carbón mineral que Chile consumía provenía casi 100\% de la producción nacional, situación que se mantendría así hasta por lo menos 1975 . Desde ese año, la producción y el consumo iniciarían trayectorias diferentes.

industrialización dirigida por el Estado (en adelante IDE). Luego de este periodo de transición se produciría la cristalización del modelo IDE entre 1945 y 1973. 
Durante este segundo ciclo, los principales sectores consumidores de carbón fueron la industria y los ferrocarriles, relegando a la minería que habría sido importante en el ciclo anterior. Ambos sectores estaban fuertemente involucrados en el proceso de industrialización por sustitución de importaciones (ISI). La industria era el sector que, por definición, estaba llamado a sustituir dichos productos; mientras que los ferrocarriles del Estado constituyeron la columna vertebral del comercio interior, tanto de las mismas manufacturas como de las materias primas (dentro de las cuales el carbón era una de las más importantes). Los trenes cargaban el carbón desde la zona sur hasta el centro del país (Santiago y Valparaíso), lugar donde se concentró la actividad industrial (Badía-Miró y Yáñez, 2015), para luego distribuir las manufacturas desde dichas ciudades hasta el resto del territorio. De esta forma, el eje Santiago-Valparaíso fue el responsable de 80\% del consumo de carbón entre los años 1939 y 1949.

Otra característica importante de este segundo ciclo es que la mayor parte del carbón mineral consumido correspondió a consumo final. Así lo demuestra el consumo de la industria fabril y de los ferrocarriles, sectores altamente intensos en consumo de carbón. Sin embargo, una parte no menor fue utilizado en la producción de gas y electricidad, y otro poco en la producción de coque metalúrgico. Proporcionalmente, cerca de 95\% del consumo total de carbón mineral de 1933 fue consumo final, cantidad que se redujo levemente hasta 1960, en que esta parte del carbón representó 86\% del consumo total. La situación cambió significativamente en 1970, cuando el consumo final de carbón representó $46 \%$ del total. Esta transición en los usos del carbón comenzó durante el segundo ciclo, y se habría resuelto específicamente en 1967, cuando la producción de energía secundaria basada en el consumo de carbón superó definitivamente al consumo final de carbón.

En las siguientes páginas abordaremos la complejidad del ciclo más contemporáneo del carbón mineral en Chile. A partir de 1973 veremos cómo a la vez que crece el consumo de carbón mineral, imponiendo su uso para la producción de energía secundaria (electricidad), la matriz energética chilena se va volviendo cada vez más compleja por la entrada de nuevas fuentes de energía primaria a la producción de electricidad (petróleo, gas natural, leña y biomasa y energías renovables) y se acentúa la dependencia energética.

\section{EL TERCER CICLO DEL CARBÓN}

El tercer ciclo del carbón es un fenómeno de las últimas cuatro décadas y aún está en desarrollo. Podríamos situar su inicio en 1973, cuando la 
inestabilidad energética generada por las crisis del petróleo cambia el ciclo energético globalmente, sin quedar Chile exento del fenómeno, para dar paso a un notorio incremento del consumo de carbón mineral desde 1986 (véase gráfica 2). Dicho aumento se realizó a una tasa de crecimiento de 7.3\% al año hasta 2013, con dos crisis en forma de U entre 1990-1997, y 1999-2008. En diez años (de 2003 a 2013), el consumo de carbón en Chile se incrementó de 2425 a poco más de 7549 KTEP. Basándonos en la información que tenemos hasta el momento, el tercer ciclo habría alcanzado su mayor nivel de consumo el año 2013, mostrando una pequeña reducción en 2014 y $2015 .^{8}$

A diferencia del segundo ciclo, en el proceso actual la producción y el consumo de carbón mineral han seguido sendas bastante diferentes entre sí, específicamente entre 1990 y 2012 . En el caso del consumo, un abrupto incremento se produjo desde 1986 -cuando las cifras reportaban la cantidad de 1324 KTEP- hasta 1999 cuando se alcanzó 4157 KTEP. Desde 1999 hasta 2003 se observan agudas variaciones que redujeron el consumo de carbón a la cantidad de 2429 KTEP, para luego incrementar de nuevo sin parar hasta 2013, cuando se consumieron 7549 KTEP. Por el contrario, hasta 1990, la producción de carbón chileno se mantuvo en niveles cercanos a los del consumo, excepto durante el periodo 1976-1987, en el que se produjo una serie de trastornos que da cuenta de la inestabilidad económica de la época, que incluye la década de la deuda externa. La mejor cifra de producción se registró en 1990, con 1848 KTEP, para luego iniciar una frenética reducción que provocaría una notoria divergencia entre esta y el consumo. La disminución señalada se realizaría hasta registrar una de las peores cifras en la historia de la producción carbonífera chilena, alcanzando niveles incluso inferiores a lo registrado en 1865 . Entre 1999 y 2012, la producción nacional promedió un total de 289 KTEP de carbón, similar a lo producido en 1872. Durante el año 2004, esta llegó a ser de 133 KTEP, y sólo 127 KTEP en 2007, icasi lo mismo que se registró en 1860! Es decir, la producción de la minería del carbón en Chile había caído en un abandono casi absoluto durante la última década. Esta situación cambiaría de forma radical en el año 2013, cuando la producción de carbón chileno se elevó a 1524 KTEP, y a más de 3000 KTEP en 2014. Este vertiginoso cambio se explica por la puesta en funcionamiento de la mina de carbón Invierno, ubicada en isla Riesco en la región de Magallanes, que ha superado todas las expectativas de producción en la historia de Chile. Sin embargo, tal

${ }^{8}$ Según los datos publicados por la Comisión Nacional de Energía (2016a), el consumo de carbón de los sistemas interconectados Central y del Norte Grande en 2015 fue casi de 1000 toneladas de carbón menos que lo consumido en 2013. Sin embargo, hasta septiembre de 2016, el consumo acumulado fue aproximadamente 131 KTEP menos que lo consumido hasta septiembre de 2013. 
dG.LY

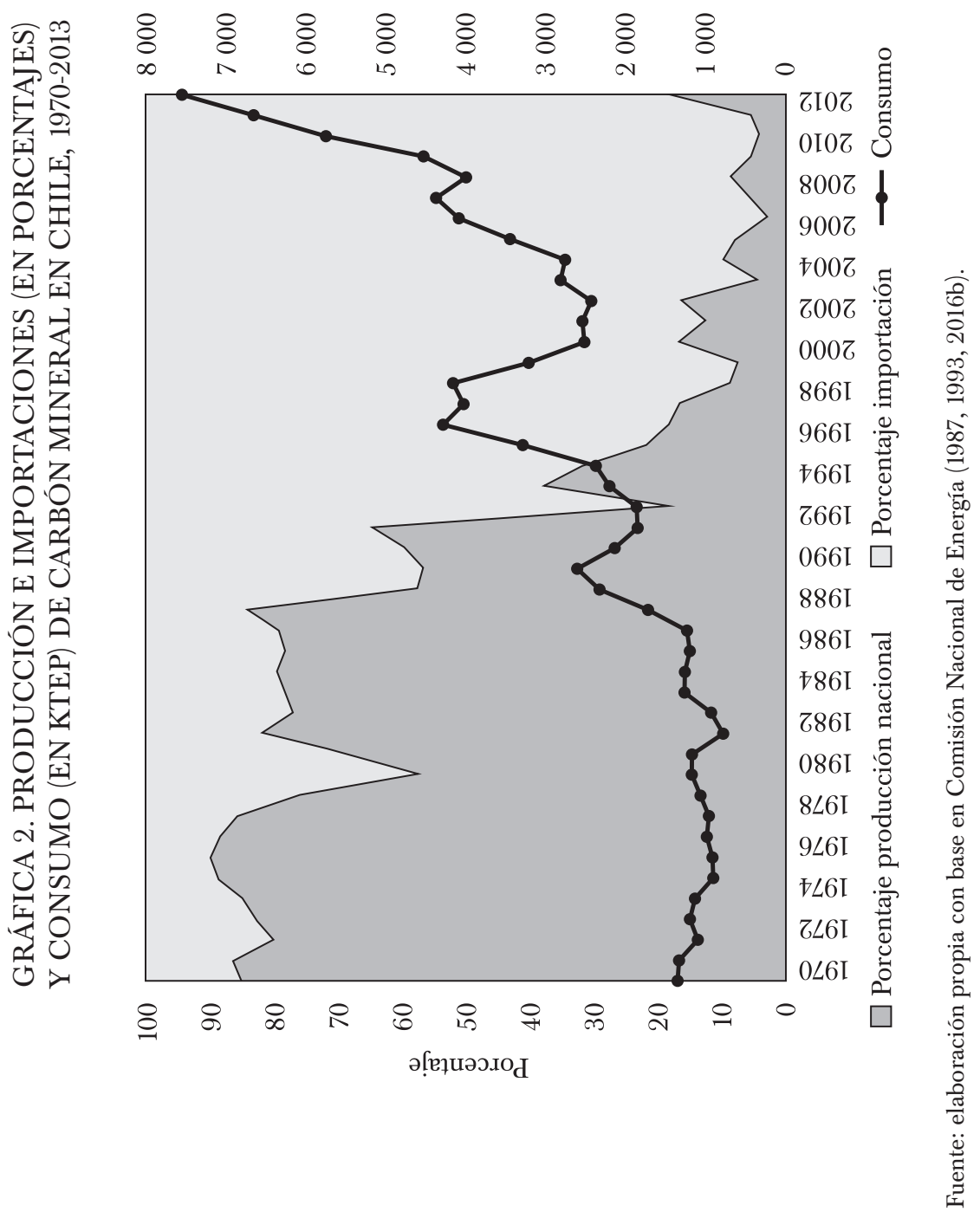


como se detallará más abajo, la reducción del precio del carbón internacional impulsó a la mina Invierno a reducir su producción a 2300000 toneladas para 2016.

Mientras esto ocurría con la producción, el abastecimiento del consumo se realizaba casi en su totalidad mediante la importación. Desde 1990, esta supuso 82\% del consumo total de carbón en Chile, elevándose a $92.1 \%$ entre 1999 y 2012. Si bien la producción de la mina Invierno ha planteado un nuevo escenario para el abastecimiento energético nacional, esto no se tradujo en una reducción significativa de la dependencia sobre las importaciones. No toda la producción de esta ha sido consumida por el mercado interno, pues $50 \%$ ha sido destinada a la exportación. De esta manera, en 2013 las importaciones de carbón seguían representando 86\% del total consumido.

\section{La transición en los usos del carbón}

Lo más significativo del tercer ciclo es la creciente tendencia a la concentración del consumo de carbón mineral en los centros de generación de electricidad (véase cuadro 2). Desde 1993, esta actividad representa más de $50 \%$ del consumo total de carbón en Chile, cifra que se incrementa a 92.7\% en 2013. Sin embargo, en 1960 el consumo de los centros de transformación a electricidad representaba 5.1\% del consumo total, y se mantuvo bajo 30\% por lo menos hasta 1977 . Si a este indicador le añadimos lo consumido por los centros de transformación de gas y coque, ambas actividades representan más de 50\% del consumo total de carbón desde 1967. Dichas actividades se caracterizan por utilizar el consumo de carbón mineral como energía primaria para la transformación en energía secundaria. La concentración del consumo de carbón en estas dos actividades, y luego, desde 1990, mayoritariamente en la generación de electricidad, es una consecuencia directa del proceso de transición en los usos del carbón mineral. Este proceso se habría iniciado en Chile a fines del segundo ciclo, específicamente entre 1961 y 1971.

Hasta 1971, el consumo final de carbón (concentrado en algunas industrias y en los ferrocarriles) tendió a reducirse significativamente, sin que esto representase una disminución excesiva del consumo total de carbón. Esto se debió a que, mientras las actividades mencionadas reducían su consumo, otras lo incrementaban, compensando la pérdida señalada. Entre estas otras actividades, las más destacadas fueron las dedicadas a la transformación del carbón en gas y coque, y la de generación de electricidad, ambas caracterizadas por producir energía secundaria con base en el carbón mineral. 


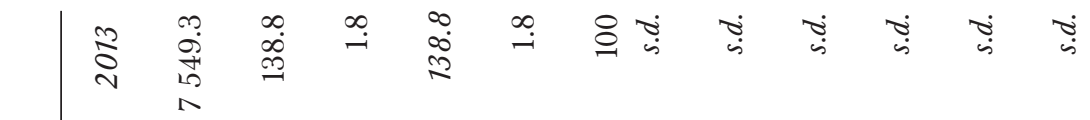

$$
\begin{aligned}
& \text { 四 }
\end{aligned}
$$

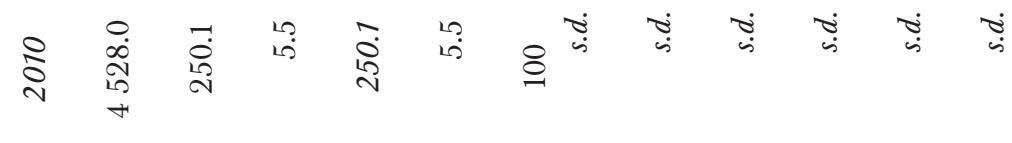

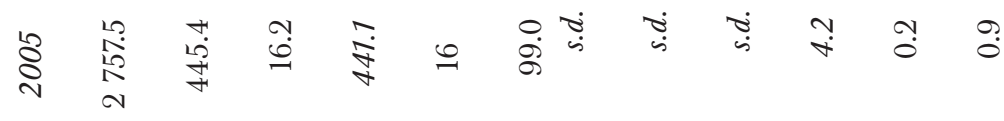

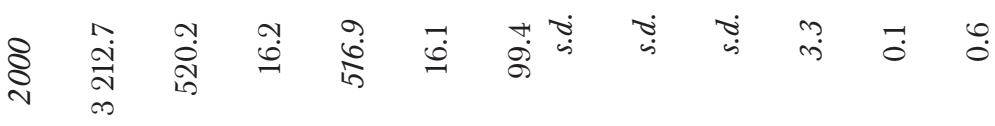

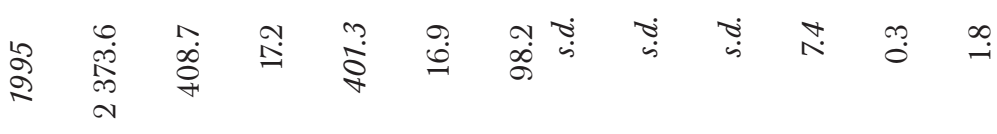

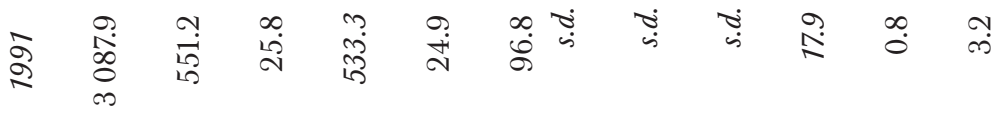

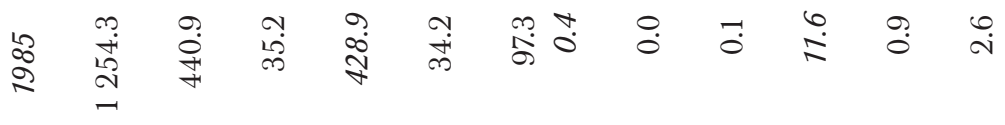

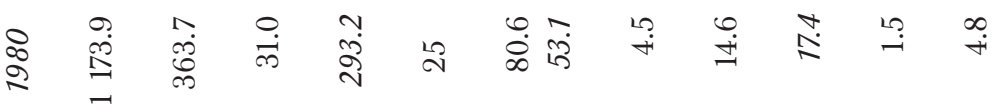

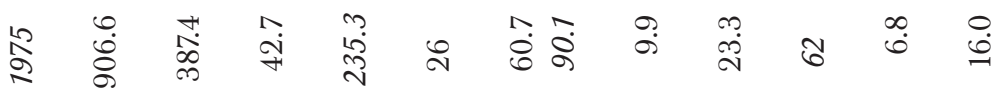

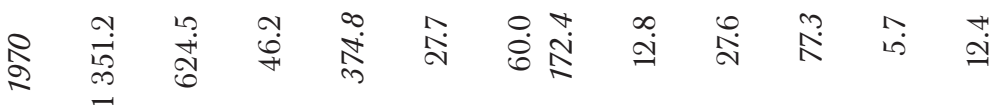

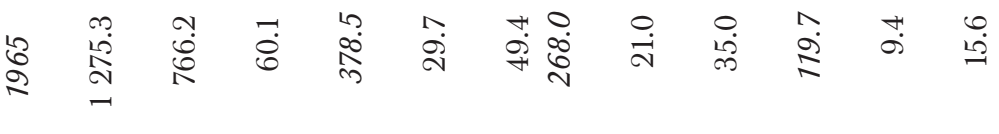

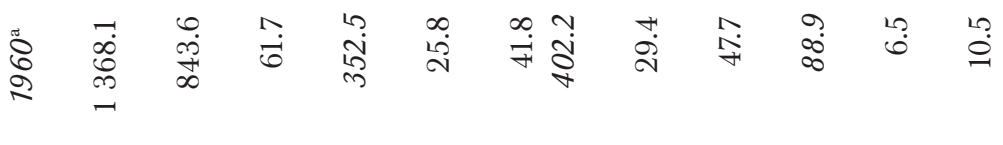

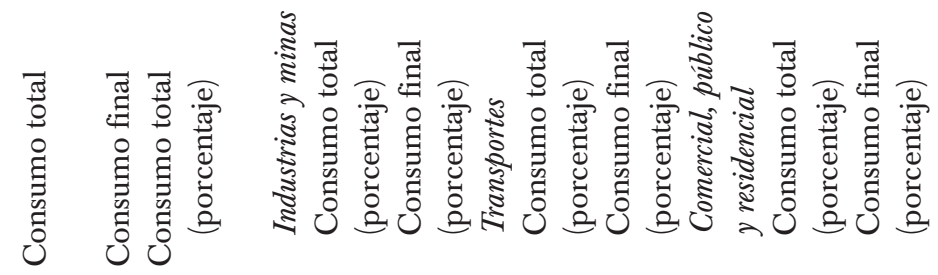




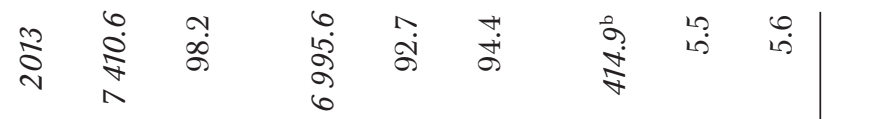

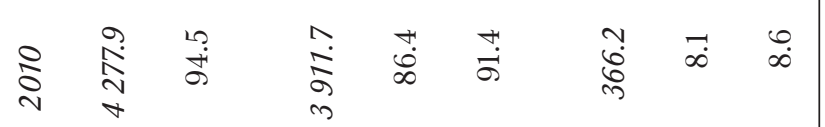

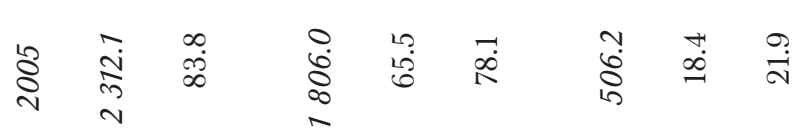

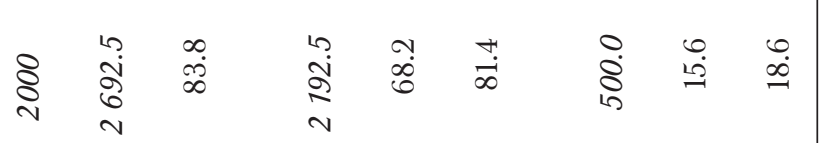

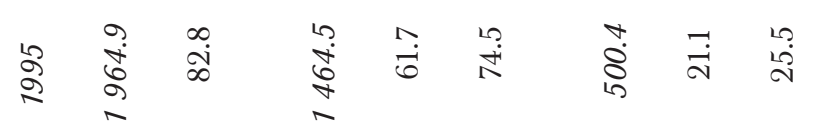

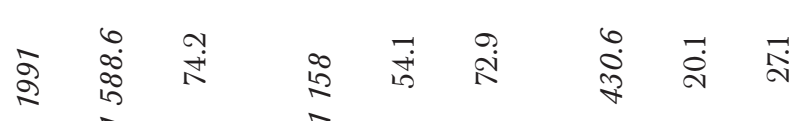

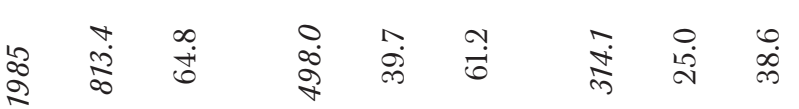

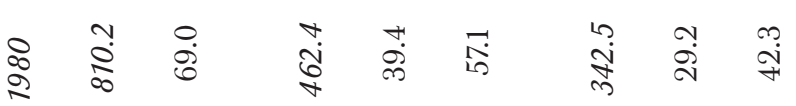

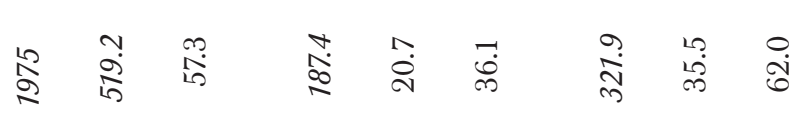

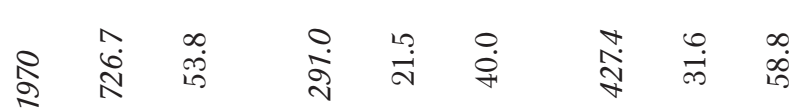

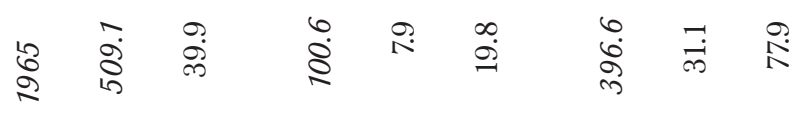

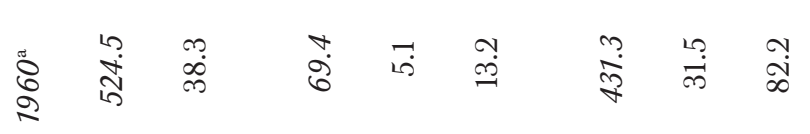

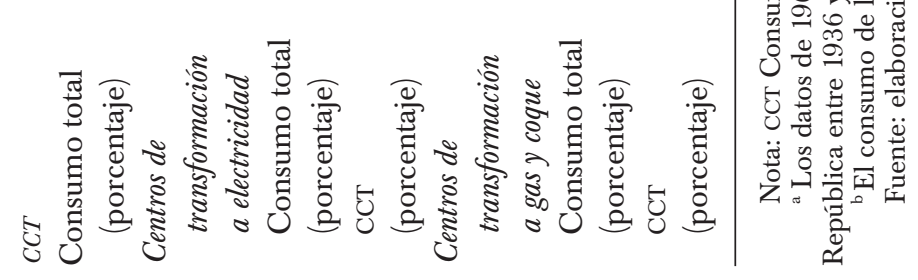

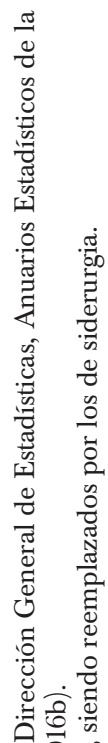

थิ จิ จ

:

峁范

헹

ํㅛㅁ응

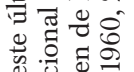

वे

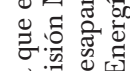

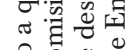

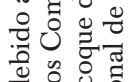

댈 $\lambda$.

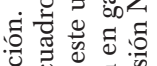

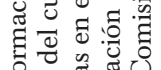

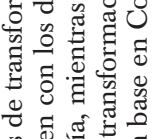

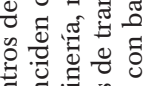

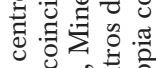

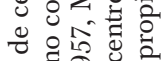

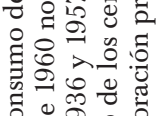


Entre 1962 y 1982, el consumo final de carbón tendió a crecer a una tasa de $-6 \%$ al año, disminuyendo desde 881 KTEP registrados en 1962 a 245 KTEP de 1982. Esta reducción no afectó por igual a todos los sectores consumidores finales de carbón, siendo los más desfavorecidos los transportes y el sector comercial, público y residencial. Durante los primeros once años de esta fase, el consumo de carbón de los transportes se redujo en más de 50\%, desde las 402.2 KTEP de 1960 a 165.6 en 1971. Finalmente registrarían 0.4 KTEP en 1985, para luego desaparecer de las estadísticas. Tal vez un caso menos abrupto pero igual de notorio fue la caída en el consumo de los sectores comercial, público y residencial, que, de consumir 130 KTEP en 1962, se desmoronaron hasta 47 KTEP en 1974, y 17.9 KTEP en 1980. Desde 1982 en adelante, el consumo de este sector no superará los 20 KTEP, salvo por una vez en 1992. En cuanto al consumo industrial y minero, su reducción es mucho menor que en el caso de los transportes y del sector comercial, público y residencial, pues desde 1960 a 1982 retrocedió 39\%, pasando de 352.5 KTEP registradas en 1960 a 217 KTEP de 1982.

Mientras disminuía el consumo final de carbón, el consumo de los centros de transformación aumentaba en 260 KTEP entre 1961 y 1971. Luego de esta etapa, las actividades transformadoras pasan por un periodo de crisis e incertidumbre entre 1972 y 1986. Pese a esta situación, el consumo de los centros de transformación nunca se redujo mucho más de lo registrado en 1960, es decir, 524.5 KTEP. Hasta 1971, el consumo de carbón utilizado en la generación de gas y coque se mantuvo estable entre 400 y 450 KTEP aproximadamente. Mientras que desde 1971 hasta 1984, el consumo de estos centros tendió a reducirse levemente hasta registrar 219 KTEP. El caso de los centros de transformación en electricidad es mucho más inestable. Durante los primeros años de esta etapa, el consumo de carbón utilizado en la generación de electricidad se cuadruplicó, incrementándose desde 69.4 KTEP en 1960 a 325 en 1971. Luego de cuatro años de crisis, el consumo de carbón se elevó de 187.4 KTEP registrados en 1975 a 566 en 1984, con una gran crisis entre 1981 y 1983. Finalmente, desde 1967, el consumo de carbón destinado a la generación de energía secundaria se habría impuesto sobre el consumo final de carbón mineral, incrementándose paulatinamente hasta 1986.

Dentro del consumo de los sectores transformadores también hubo una leve transición. Desde 1960 hasta 1978, el principal consumidor de carbón mineral en Chile fueron los centros de transformación a gas y coque y, mucho más abajo, los centros de transformación a electricidad. Sin embargo, el incremento del consumo de estos últimos, descrito con anterioridad, cambió el panorama en 1978, situando a estas industrias como el principal sector consumidor de carbón del país. Desde dicho año hasta la actualidad, el consumo de carbón se ha concentrado fuertemente en la 
transformación de electricidad, superando 98\% del consumo total de carbón durante 2013 (tal como se señaló más arriba).

\section{LA DISTRIBUCIÓN DEL CARBÓN EN EL TERCER CICLO}

Pese a la extrema concentración del consumo de carbón mineral en el sector transformador a electricidad, el consumo de carbón de las demás actividades presenta un comportamiento bastante interesante, que es necesario explicar para entender la totalidad de esta tercera etapa. Para mejorar el análisis de la distribución, dividiremos el consumo de carbón en actividades consumidoras finales de carbón y aquellas transformadoras de carbón en energía secundaria (véase cuadro 2).

\section{El consumo final de carbón}

Durante este tercer ciclo, el consumo final de carbón ha mostrado una inestabilidad constante dentro de una tendencia claramente descendente, dando cuenta de tres periodos: desde 1961 hasta 1982 tendió a crecer a un ritmo de $-5.8 \%$ al año; entre 1982 y 2001 se recupera a una tasa de crecimiento de 3.6\% al año, marcando un máximo excepcional en $1997 ;{ }^{9}$ y finalmente, volvería a crecer hasta 2013 a un ritmo de $-12.5 \%$ al año, llegando a su mínimo histórico (véase gráfica 3).

Las actividades consumidoras finales de carbón han sido agrupadas en tres sectores: industrial y minero; comercial, público y residencial, y transportes. De estos tres grupos, el que reúne a los transportes se redujo constantemente hasta desaparecer de las estadísticas desde 1985, producto de su virtual abandono y desaparición de sus funciones. En 1970 representaba más de un cuarto del consumo final de carbón, situación que se redujo, en quince años, a 0.1 por ciento.

Las actividades agrupadas en el sector comercial, público y residencial inician el tercer ciclo consumiendo 77.3 KTEP, equivalente a 12\% del consumo final. Esta cantidad se reduce fuertemente en 1980, y desde ahí lo hará en forma constante hasta desaparecer de las estadísticas en 2005, cuando consumieron $4.2 \mathrm{KTEP}$, equivalente a $0.9 \%$ del consumo final.

${ }^{9}$ La cifra registrada en 1997 muestra un comportamiento anormal al analizar la tendencia del consumo del grupo y de las partes que lo componen. Tal monto se debe exclusivamente al consumo del sector industrias y minas varias, que registró 624.6 KTEP, es decir, un promedio de más de 400 más que los años 1996 y 1998. Debido a las dudas que pueda suponer este comportamiento, se prefirió el análisis de medias móviles. 


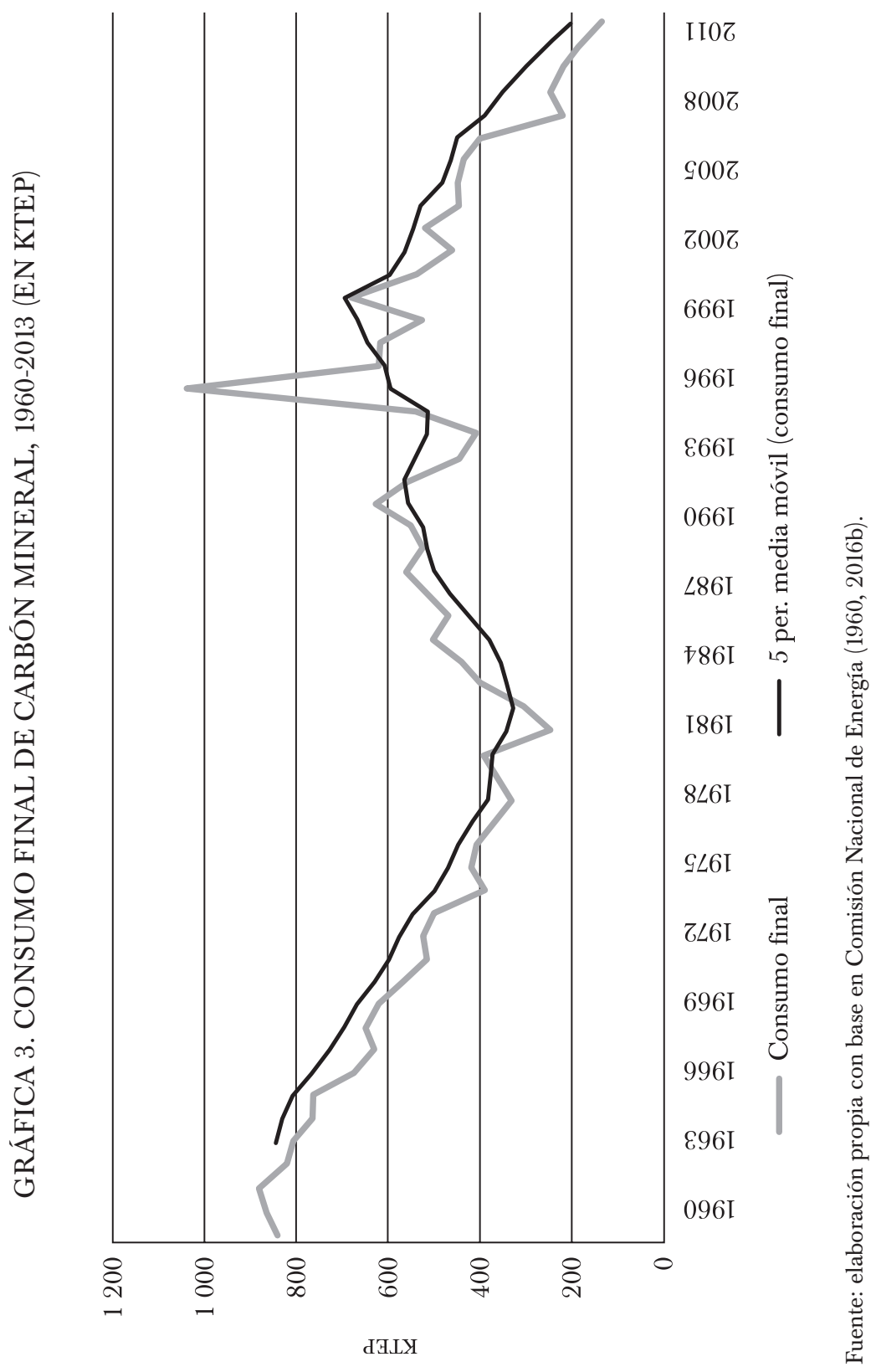


En cuanto a las actividades agrupadas en el sector industrial y minero, mantuvieron una situación bastante inestable desde los inicios del tercer ciclo, pero que se intensificó entre 1986 y 2001, año desde el que iniciaron una reducción constante del consumo de carbón mineral hasta 2013. En esos doce años, el consumo de carbón de este grupo se redujo de 685 a 138 KTEP. En promedio, la mayor parte del consumo del sector fue concentrado por un grupo denominado industrias y minas varias, del cual los balances no especifican más detalle. Su participación en el grupo de industrias y minas muestra variaciones bastante amplias durante todo el ciclo, registrando $16 \%$ en 1982, 61\% en 1997 y 18\% en 2008 (véase gráfica 4). Sin embargo, en promedio representó 32\% del consumo del grupo. Medido en unidades físicas, el consumo de las industrias y minas varias se inicia con 120 KTEP en 1970, para reducirse a 47 hasta 1983. El consumo de 1985 volvió a los niveles de la década de 1970, anotando 141 KTEP, para luego escalar a 205 en 1989, y un récord de 624 KTEP en 1997. Sin embargo, en el año 2000 volverá a marcar niveles cercanos a 150 KTEP. El año de recuperación será 2001 (365 KTEP), pero el resto del periodo describirá una reducción hasta 2013, en que consumirá 60 KTEP.

Siguiendo a las industrias y minas varias, el consumo de carbón de la industria del cemento se ubica en el segundo lugar, por lo menos hasta el año 2008, desde el cual se reduce hasta casi desaparecer del registro, consumiendo sólo 0.1 KTEP en 2013. El consumo de carbón de esta actividad pasa por un periodo de inestabilidad entre 1970 y 1990, para luego iniciar una trayectoria ascendente con forma de dientes de sierra, entre 1990 y 2008. Su consumo promedio durante este ciclo fue de 115 KTEP, con un mínimo de 62.3 en 1976 (sin considerar la virtual desaparición de los últimos cuatro años), y un máximo de 214 KTEP en 2008. En 2009 y 2010 registrará 51 y 53 KTEP, respectivamente, para luego reducirse a un KTEP en 2011, y 0.1 en 2013. Un poco más abajo se encuentra la industria azucarera, que inició el periodo consumiendo $93 \mathrm{KTEP}$. Al igual que la industria del cemento, muestra un periodo de crisis entre 1970 y 1990, con una caída de más de 70 KTEP entre 1976 y 1982 . Entre 1986 y 2002 mantendrá niveles casi constantes que fluctúan cerca de los 100 KTEP. Desde 2003 iniciará un ciclo negativo hasta 2001, en que se reducirá su consumo en casi 60 KTEP. Finalmente, en 2013 registrará un consumo de 69 KTEP.

La industria pesquera figurará en las estadísticas de los balances energéticos recién desde 1991. Su trayectoria muestra una tendencia a la reducción del consumo de carbón, que se verá interrumpida por dos periodos de recuperación. Su máximo consumo se registró en 1991, con 85 KTEP, y se redujo a 32 en 1995. En 1996 se mostrará la primera recuperación, que durará dos años, para luego reducirse a diez KTEP en 2000. Desde 2000 hasta 2013, promediará 8.5 KTEP con un máximo de 26 en 2004. 


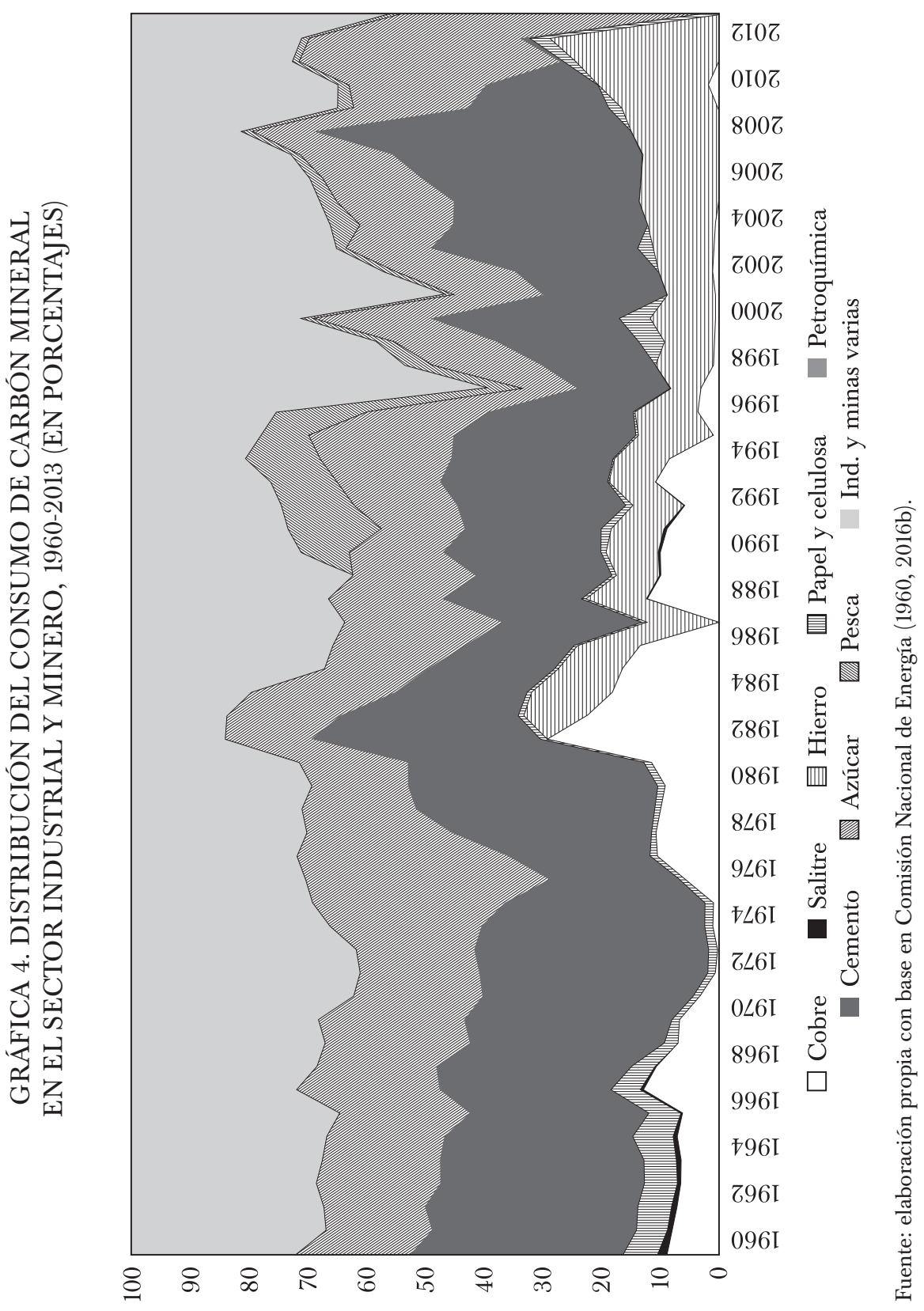


El consumo de carbón de la gran minería chilena se caracteriza, durante este tercer ciclo, por su abandono casi absoluto. En cuanto a la industria salitrera, que fuera uno de los más grandes consumidores de carbón a principios del siglo XX, registra consumo durante cuatro años de este ciclo (1991-1994), promediando 1.2 KTEP por año. Muy diferente es el caso de la minería del cobre, que inicia el periodo con un incremento sustancial de dos KTEP que consumía en 1975, a casi 70 de 1985. Dicha cantidad se reducirá en forma constante hasta el año 2000, cuando registró un consumo de 2.5 KTEP. Entre 2000 y 2010, promediará 2.3 KTEP de carbón al año, alcanzando el nivel marginal antes señalado, para desaparecer de las estadísticas desde 2010 en adelante.

Un consumo igual de reducido es el que muestra la industria del papel y la celulosa que, durante todo el periodo (1970-2013), promedió un total de cuatro KTEP al año, registrando sus mejores cifras en 1999 y 2000, con 27 KTEP cada año. El sector se decidió muy temprano por el petróleo como fuente de energía y después por una sorprendente combinación de leña y electricidad. En cuanto a la industria del hierro, su consumo de carbón muestra una oscilación constante durante todo el periodo, que variará entre 35 y 60 KTEP entre 1983 y 2012.

\section{El consumo de carbón para la energía secundaria}

El grupo de los centros transformadores de carbón en energía secundaria se compone por los centros de transformación a electricidad, a gas y coque, a carbón y leña y, desde 2012, de la siderurgia. Los balances energéticos registran los datos del consumo de carbón de los centros transformadores en carbón y leña hasta 1986, debido a la baja cantidad de su consumo. En 1986, el consumo de estos centros fue de $1.8 \mathrm{KTEP}$, mientras que, en promedio, entre 1966 y 1986, consumieron 6.6 KTEP (véase gráfica 5).

El consumo de carbón mineral de los centros de transformación en gas y coque durante las últimas décadas del segundo ciclo fue explicado más arriba, en el contexto de la transición en los usos del carbón (véase cuadro 2). Este sector parte el tercer ciclo consumiendo 427.4 KTEP, para reducirse a 321.9 en 1975. Desde aquí se incrementará hasta alcanzar 500 KTEP en 1995, y mantener este promedio durante los siguientes diez años. Entre 2008 y 2010 se observa una ligera variación negativa que reducirá el consumo de carbón hasta 366 KTEP para volver a los valores cercanos a 500 en 2011. En 2012, estos centros han desaparecido de las estadísticas de los balances energéticos. Sin embargo, 2012 será el primer año en que figurará el consumo de carbón de la siderurgia. Las cifras registradas por esta nueva actividad son ligeramente inferiores al último consumo de los 


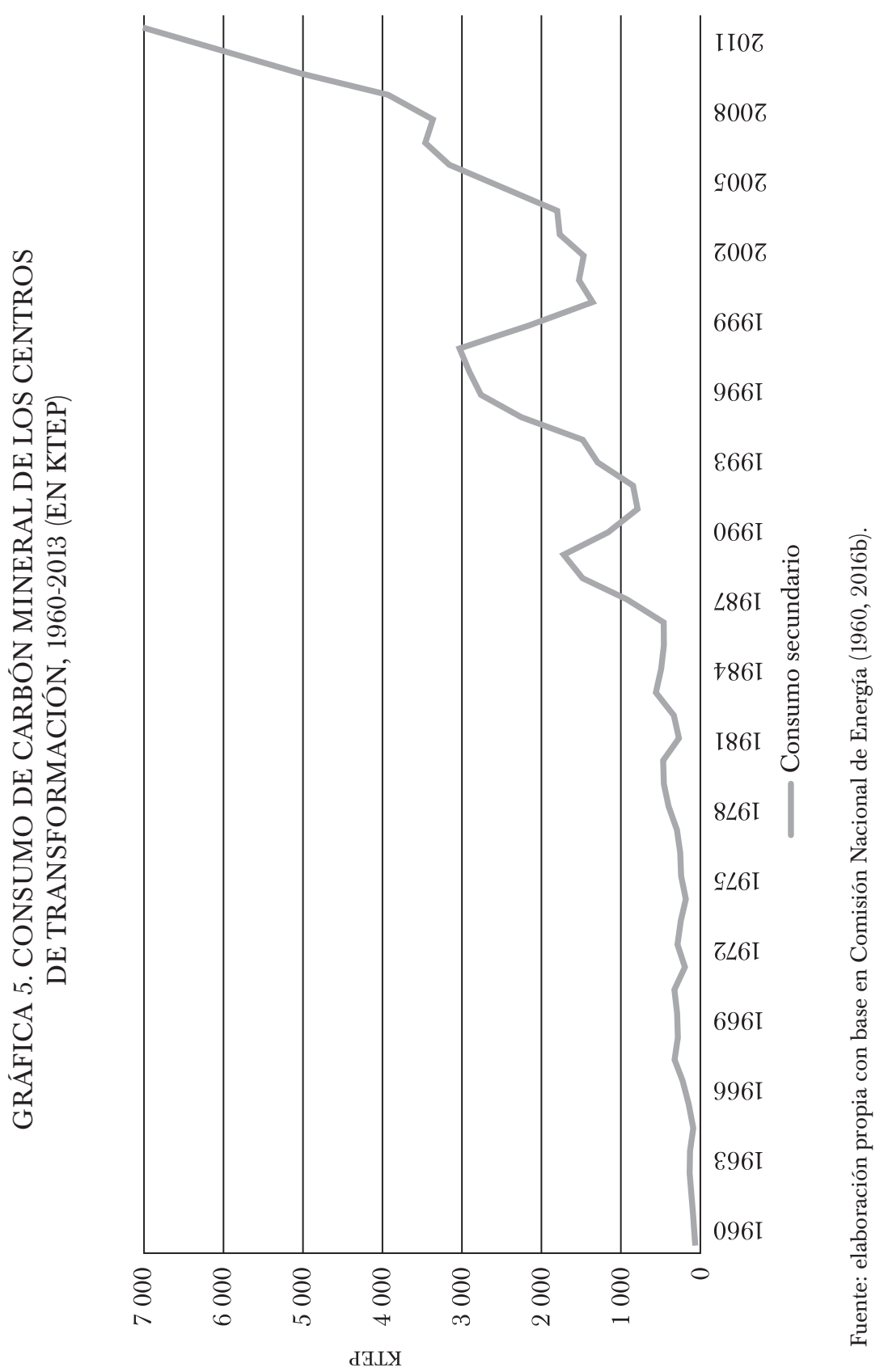


centros de gas y coque, razón por la cual suponemos que la siderurgia es el consumo de carbón utilizado para transformar en coque, contenido en la nomenclatura anterior.

Proporcionalmente, y como se señaló en el apartado anterior, los centros de generación de gas y coque representaron más de $80 \%$ del consumo de carbón destinado a la generación de energía secundaria en 1960, situación que fue cambiando durante fines del segundo ciclo, hasta ser completamente superado por el consumo de los centros de generación de electricidad en 1978. En 1986, su representación se reducía aún más hasta bordear 40\%. Finalmente, en 2011 representarían 8.8 y $5.6 \%$ en 2013, si consideramos el consumo de la siderurgia como la continuación de esta actividad.

El segundo exponente del consumo de carbón mineral para la generación de energía secundaria son los centros de transformación a electricidad (un análisis más acabado sobre la importancia de otras fuentes de energía se presenta más adelante). Estos iniciaron el tercer ciclo del carbón con un consumo de $291 \mathrm{KTEP}$, cantidad que se incrementa notoriamente en 1991, cuando registraron 1158 KTEP. Luego de una pequeña crisis entre 1991 y 1994, el consumo de carbón de este sector vuelve a elevarse hasta 3038 KTEP registrados en 1999. Sin embargo, en dos años, el avance logrado hasta ese entonces se redujo a 1334 KTEP en 2001.

Hasta 2005 habrá un pequeño aumento de 500 KTEP que, pese a ser limitado en relación con las cantidades consumidas por esta actividad, representa mucho más de lo que cualquier otra industria consumía dicho año, incluso más que todas las actividades consumidoras finales de carbón en conjunto. Pero el consumo volvería a crecer una vez más, hasta superar incluso la marca registrada en 1997. Desde 2005 se iniciará un incremento descomunal del consumo de carbón, con un crecimiento de 18.4\% al año, hasta registrar casi 7000 KTEP de 2013. Gracias a las publicaciones de la Comisión Nacional de Energía (2016b), hemos podido conocer el consumo de carbón tanto del Sistema Interconectado Central, como del Sistema Interconectado del Norte Grande (en adelante SING) para el año 2014 y hasta septiembre de 2015. ${ }^{10}$ Pese a que las fuentes son diferentes, hemos podido constatar que el año 2014 se consumió cerca de 400 KTEP menos que en 2013. Y hasta septiembre de 2015 se consumió cerca de 140 KTEP menos que en el mismo periodo del año anterior. Algo cambió en el sector, lo que podría anunciar la apertura de una cuarta etapa, aunque esto aún es pura hipótesis.

${ }^{10}$ Los sistemas interconectados Central y del Norte Grande agrupan la mayor parte de la generación de electricidad en Chile, correspondiente a 95\% del total generado en 2010. Al conocer el consumo de energía de estos sistemas, y salvo que haya ocurrido algo absolutamente inesperado con el consumo final, podemos conocer más de $98 \%$ del consumo total de carbón. 


\section{EL CARBÓN MINERAL Y LA ELECTRICIDAD}

Desde 1930 y hasta antes de los choques del petróleo, la mayor parte de la generación termoeléctrica latinoamericana se realizaba con base en el consumo de petróleo. El sustancial incremento de los precios del oro negro puso fin a esta situación, dando inicio a una nueva era de la generación eléctrica, en la que la hidroelectricidad parecía ser la alternativa más utilizada (Rubio y Tafunell, 2014). La termoelectricidad, en cambio, se caracterizó por abrirse al consumo de otras energías primarias que sustituyeran al petróleo. Los efectos de la crisis petrolera se hicieron sentir en Chile, reduciendo notoriamente el consumo de petróleo destinado a la generación de electricidad. ${ }^{11}$ El escenario generado fue sorteado por la Empresa Nacional de Electricidad, S. A. (en adelante ENDESA) con el aumento de la capacidad instalada de las centrales hidroeléctricas, pero también las termoeléctricas de carbón. Por lo menos hasta 1978, el petróleo era el principal combustible utilizado en la generación de termoelectricidad; sin embargo, en 1991 el carbón había alcanzado el primer lugar del consumo energético de estos centros.

La crisis del petróleo coincide con un momento en que un importante proceso modernizador se estaba desarrollando en Chile, lo que incrementó sustancialmente la demanda eléctrica nacional. Hasta 1946, más de 50\% de la generación eléctrica nacional fue destinada a abastecer la demanda de la gran minería (véase cuadro 3). Pese a incrementar constantemente su consumo, la participación porcentual de la minería en el consumo final de energía eléctrica en Chile se reduciría sin parar hasta 1985. Desde este año hasta fines del periodo estudiado, la minería volvería a recuperar su posición, incrementado su participación en el consumo final de electricidad de 24.9 en 1985 a 33.2\% en 2010. Entre 1960 y 1985, un importante proceso modernizador incrementó notablemente la demanda eléctrica de otros sectores. Una parte importante de este incremento fue concentrado por las industrias manufactureras, que comenzaron a incorporar tecnología moderna una vez finalizada la segunda guerra mundial (véase Yáñez y Garrido, 2015, cuadro 6). Sin embargo, la modernización no se concentró

${ }^{11}$ Ya en 1980, y muy influenciado por las crisis del petróleo, Bruno Philippi (1980) señalaba la necesidad de reducir el consumo de petróleo en el país, y daba cuenta de algunos planes políticos para lograr este objetivo. Entre ellos, señalaba la puesta en funcionamiento de una línea eléctrica entre Santiago-Huasco-Chañaral que permitiría sustituir una parte importante del consumo de petróleo para la generación de electricidad en el norte, por hidroelectricidad y por generación a carbón. La importancia que el autor le otorga al carbón es tal que, según él, el carbón permitiría una independencia total (sic) del país respecto al petróleo. 


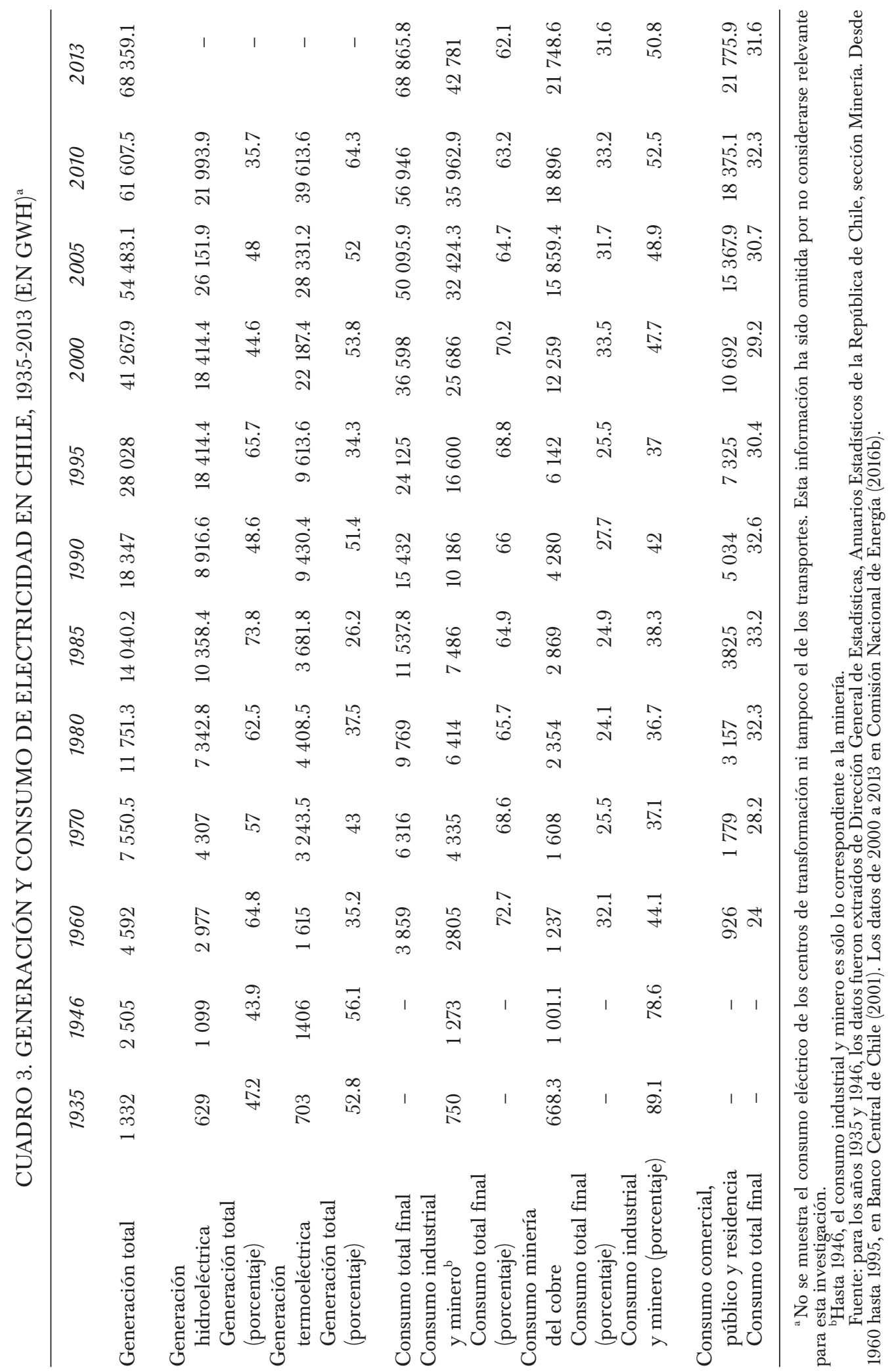


solamente en estos centros productivos, sino que incorporó un nuevo elemento: la urbanización. ${ }^{12}$

La industrialización dirigida por el Estado fomentó la migración campo-ciudad, incrementando el número de la población urbana en Chile. ${ }^{13}$ Desde 1940, esta fracción de la población comenzó a ser mayoritaria en la estructura demográfica chilena, fomentando la demanda de bienes y servicios, caracterizados por el consumo de electricidad. Los incrementos en las tasas de urbanización han sido reconocidos por provocar una mayor demanda energética, especialmente de electricidad (Rubio y Tafunell, 2014). Esta situación no es ajena al caso chileno pues, entre 1960 y 1980, el crecimiento del consumo eléctrico del sector comercial, público y residencial se incrementó a 6.3\% al año, aumentando de 926 a 3157 Gwh en dicho periodo. Entre estos años, el crecimiento del consumo eléctrico de las industrias y minas se realizó a 5\%, mientras que el consumo final de electricidad lo hizo a 4.8\%. Desde 1985 hasta 2013, el consumo del sector comercial, público y residencial creció a una tasa de $6.4 \%$ al año, mientras que el sector de industrias y minas lo hizo a un ritmo de 5.1\%, y el consumo final de electricidad a $4.8 \%$ al año. Este proceso modernizador del sector comercial, público y residencial se expresa, también, en su participación porcentual sobre el total de consumo final de electricidad, aumentando de 24 en 1960 a 33.2\% en 1985. De esta forma, entre 1960 y 1985, el consumo final de electricidad casi se habría triplicado, presionando a ENDESA a promover la generación de electricidad para abastecer la creciente demanda.

Para responder a las necesidades eléctricas identificadas desde temprano en la economía chilena, ${ }^{14}$ ENDESA llevó a cabo una serie de planes

${ }^{12}$ Donald Jones (1991) sugiere que la urbanización es un problema energético aún mayor que la industrialización pues, pese a que están asociadas, no son idénticas en sus características energéticas. La industrialización permite identificar formas de reducir el consumo energético, tales como la renovación de equipos, sustitución de combustibles, etc., mientras que la urbanización presenta mayores complejidades en esta materia.

${ }^{13}$ Hasta 1930, la población rural en Chile representaba 51\% del total de habitantes. Diez años después esta cantidad se había reducido a 47\%, producto de un incremento del número de habitantes en zonas urbanas. Entre 1940 y 1982, la tasa de crecimiento de la población urbana aumentó a 3.85 al año, mientras que la rural creció a $-0.5 \%$ al año (Economic History \& Cliometrics Lab-Iniciativa Científica Milenio. Recuperado de http://www.economia.puc.cl/cliolab).

${ }^{14}$ Sobre las dificultades por las que atravesaba la generación eléctrica en Chile, véase (Corporación de Fomento de la Producción, -CORFO-, 1939). En cuanto a las consecuencias de la falta de suministro eléctrico: "hay industrias que no pueden funcionar debidamente, a causa del servicio deficiente que se les proporciona; otras, no se instalan por la imposibilidad de adquirir la energía motriz que necesitan; el estándar de vida de los centros poblados se mantiene estacionario en lo que se refiere al alumbrado y consumo residenciales por las tarifas restrictivas impuestas por las Empresas Eléctricas como consecuencia de su falta de potencia" (CORFO, 1939, pp. 10 y 11). Las sugerencias realizadas por el cuerpo de ingenieros fueron utilizadas por la Corporación de Fomento de la Producción como la base del Plan de Electrificación Nacional, aprobado en 1942, e implementado en las décadas siguientes por la Empresa Nacional de Electricidad, S. A. (en adelante ENDESA). 
de electrificación nacional, consistente en desarrollar sistemas de abastecimiento regionales, interconectarlos y finalmente construir grandes centrales que abastecieran los sistemas antes implementados. ${ }^{15}$ De estos tres planes, es el último, desarrollado entre 1968 y 1985, el que mayor relevancia tuvo para nuestro estudio, y que coincide con el fin del segundo ciclo del carbón y el inicio del tercero. Dicho plan estuvo basado en el desarrollo de grandes proyectos de generación y transmisión de electricidad, dirigidos por la empresa estatal, al que se sumaron otras empresas del sector privado que prestaban servicio a ENDESA.

Entre los grandes proyectos generadores de electricidad construidos en esta etapa se encuentran algunos de las más importantes centrales termoeléctricas consumidoras de carbón del país. Entre estas centrales, aquella que habría inaugurado el ciclo corresponde a la central termoeléctrica Ventanas, que inició sus funciones en 1964 en la zona central de Chile. Ventanas fue, en su momento, la generadora a carbón de mayor potencia instalada en el país, con 118 Mw. Luego de Ventanas, ENDESA inauguró en 1970 la central Bocamina, ubicada en Coronel, que aportó 125 Mw de potencia en el sur. A estos proyectos se sumaría el de la central termoeléctrica Tocopilla, que desde 1987 aportaría con $440 \mathrm{Mw}$ de potencia al SING (Instituto de Ingenieros de Chile, 1988, p. 79). Casi una década después, la misma empresa habría inaugurado una nueva central termoeléctrica en Mejillones, bautizada con el mismo nombre de su emplazamiento, que aportaría $591 \mathrm{Mw}$ de potencia también en el norte. Hasta 1995 no se construirían más centrales termoeléctricas a carbón en Chile.

Sin embargo, y pese a la magnitud de las centrales señaladas, el incremento en el consumo de carbón no se produjo inmediatamente. La mayor parte de la potencia instalada durante esta fase de desarrollo se basó en la hidroelectricidad, siendo este el periodo en el que se construyeron las principales centrales hidroeléctricas que abastecen el Sistema Interconectado Central. Dentro de este grupo se encuentran Rapel (con $350 \mathrm{Mw}$ desde 1968), El Toro (con $400 \mathrm{Mw}$ desde 1973), Antuco (con $324 \mathrm{Mw}$ desde 1981) y Colbún (con $478 \mathrm{Mw}$ desde 1985). De esta forma, en 1985, de $3967 \mathrm{Mw}$ de potencia instalados en el país, $2266 \mathrm{Mw}$ correspondían a centrales hidroeléctricas y $1700 \mathrm{Mw}$ a centrales termoeléctricas.

${ }^{15}$ El plan de electrificación nacional que implementaría ENDESA comprendía tres etapas a desarrollar en 18 años. En la primera etapa se desarrollarían sistemas regionales de abastecimiento local. En la segunda etapa se pretendía interconectar los sistemas regionales, con el fin de transmitir los excedentes desde una región a otra. Finalmente, la tercera etapa pretendía por un lado la construcción de grandes centrales que, aprovechando las ventajas estacionales de las diversas regiones, transmitieran la energía hacia el norte o hacia el sur según fuese necesario, y por otro, operar de forma eficiente y económica todos los sistemas bajo la dirección de un solo comando. Las dos primeras etapas fueron finalizadas recién en 1960, mientras que la tercera se iniciaría en 1968 (ENDESA, 1993). 
La tercera etapa del plan de electrificación de ENDESA coincidió, además, con la tercera fase de las reformas de mercado implementadas por la dictadura. ${ }^{16}$ De acuerdo con el Instituto de Ingenieros de Chile (1988, p. 25), existió una crisis en los precios de la electricidad, desarrollada en el periodo 1970-1973, debido al manejo político de las tarifas que se tradujo en una prohibición para reajustar los precios; el Estado asumió los costos asociados a la inflación del periodo. El estancamiento de las tarifas generó mayor demanda eléctrica, lo que provocó un desfinanciamiento de las empresas. Para paliar la situación de desfinanciamiento, el régimen inició una serie de reformas que afectó directamente al sector público. Según Óscar Muñoz Gomá (1995), entre las reformas aplicadas en el marco del tratamiento de shock, se encuentra una reforma fiscal drástica, en la que se aplicó un grupo de medidas orientadas a reformar el sector público. Entre estas medidas se cuenta un incremento sustancial de los precios y tarifas cobradas por las empresas eléctricas que permanecieron en control del Estado. Según Larraín (1990, en Muñoz, 1995), las tarifas eléctricas se multiplicaron por diez en esos años (Muñoz, 1995, p. 29). Muy probablemente, el incremento de las tarifas eléctricas estimuló al sector privado para controlar a las empresas generadoras de electricidad. Dicha privatización se habría iniciado, en el caso de ENDESA, en 1986. Hacia 1990, cerca de 96\% de la empresa se encontraba privatizada (Meller, 1996, p. 269). ${ }^{17}$

\section{LAS CRISIS DE ABASTECIMIENTO ENERGÉTICO}

La mayor parte de los inusitados cambios en el consumo de carbón de los centros de transformación a electricidad que ocurrieron durante este tercer ciclo han coincidido con diversas crisis energéticas a nivel nacional (véase gráfica 6). Este es el caso de los incrementos registrados entre 1987-1991,

\footnotetext{
${ }^{16}$ Esta fase se caracterizó por presentar un enfoque de economía cerrada y basarse en un aumento transitorio de los aranceles a las importaciones, la administración centralizada del crédito externo, destinado a renegociar la deuda externa, devaluación real destinada a promover exportaciones y en una nueva política de restricciones salariales y de gasto público (Muñoz, 1995, p. 28).

${ }^{17}$ Entre los cambios institucionales implementados en dictadura, uno significativo fue la creación de la Comisión Nacional de Energía en 1978. Esta sería la antesala de la privatización de ENDESA, debido a que la nueva institución asumía las tareas de planificación que antes habían correspondido a la empresa estatal, limitando a esta a las funciones generadoras, transmisoras y distribuidoras, facilitando de esta manera su privatización (ENDESA, 1993, p. 93). Los efectos de las privatizaciones en la matriz energética chilena aún no han sido profundamente analizados, ni tampoco el impacto de las políticas públicas de los últimos 25 años. Una tarea pendiente es comparar el desempeño del sector energético bajo la mirada de los últimos grandes periodos de la política chilena, cuestión que ayudaría en la discusión sobre el papel del Estado y su importancia en áreas estratégicas, como es la energía.
} 


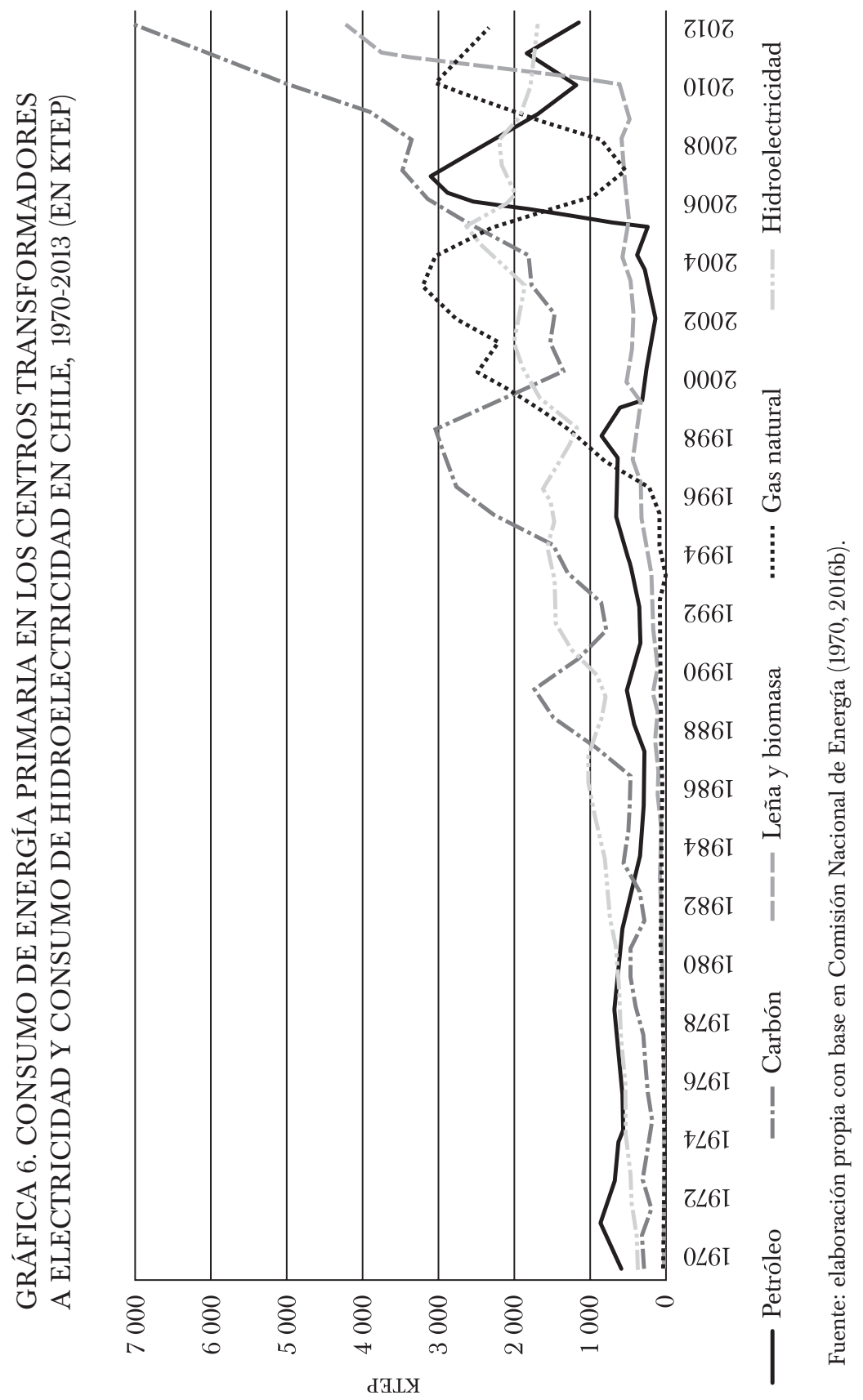


1993-1999 y 2005-2013 que hemos señalado anteriormente. Estas tres grandes variaciones del consumo de carbón han ocurrido en momentos en que la generación de electricidad ha sufrido diversas crisis que le han impedido responder con normalidad a la demanda local. De esta forma, el carbón mineral se ha convertido en una alternativa cuando otras fuentes primarias sufren adversidades que les impiden responder adecuadamente a las necesidades de los centros de transformación.

$\mathrm{Al}$ comienzo de este tercer ciclo, una grave crisis afectó la generación hidroeléctrica nacional, reduciendo de 12104 Gwh generados en 1987, a 8916 de 1990 (véase cuadro 3). En el mismo periodo, la generación termoeléctrica aumentó de 3531 a $9430 \mathrm{Gwh}$, compensando la pérdida ocasionada por la hidroelectricidad. Los datos con los que contamos hasta el momento no nos permiten saber exactamente cuánto de este aumento correspondió a generación basada en el consumo de carbón; sin embargo, sabemos que entre 1987 y 1990 el consumo de carbón de los centros generadores de electricidad se impuso por sobre las otras tres energías primarias utilizadas en la generación térmica (petróleo, gas y leña). De este modo, de toda la energía primaria utilizada en 1991 en los centros de transformación de electricidad, $67 \%$ correspondió a consumo de carbón, $22.8 \%$ a petróleo, $4.4 \%$ a gas y $5.8 \%$ a leña y biomasa. Este incremento en el uso del carbón para la generación de electricidad duró cuatro años, pues la recuperación de la hidroelectricidad provocó una reducción notoria del carbón aplicado a esta actividad. Una situación similar habría sucedido entre 1993 y 1999, cuando una nueva crisis en la hidroelectricidad habría impulsado un incremento en el uso del carbón para la generación eléctrica. Al parecer, los conflictos hidroeléctricos motivaron a las empresas a elevar la generación termoeléctrica con base en carbón, situación que se habría reducido desde 1999, cuando hizo su entrada el gas natural. Desde 1997 hasta 2004, el consumo de gas natural en los centros de transformación a electricidad se elevó de 183.7 a 3225 KTEP, mientras el consumo de carbón se redujo de 3038 en 1999 a 1336 KTEP en 2001.

El panorama parecía alentador; sin embargo, una abrupta reducción en el abastecimiento de gas ocurrido en 2006 hizo caer su consumo a 527 KTEP en 2008. Para paliar esta crisis, las empresas recurrieron nuevamente al carbón mineral. De esta forma, entre 2005 y 2009, el consumo de carbón se habría incrementado nuevamente de 1806 a 3484 KTEP, absorbiendo el vacío dejado por la generación eléctrica con base en gas natural. Durante el mismo periodo (2006-2008), el consumo de petróleo de estos centros se habría elevado de 258.9 a 2950 KTEP, justamente para ayudar a solventar la crisis provocada por la reducción del gas natural. Sin embargo, desde 2009 se iniciaría un nuevo conflicto de abastecimiento de 
energías primarias, pero esta vez afectando al petróleo, que reduciría su consumo a 1205 KTEP en 2013.

A este nuevo escenario, caracterizado por conflictos en el abastecimiento de gas y petróleo, se sumó una de las peores sequías que haya afectado a la zona central de Chile. La cruda reducción del nivel en los embalses afectó notoriamente la generación hidroeléctrica, reduciendo su producción de poco más de 26000 Gwh generados en 2005, a casi 22000 en 2010. Los centros de transformación a electricidad buscarían nuevamente la solución en el consumo de carbón, que se habría incrementado desde 3350 KTEP en 2009 a casi 7000 en 2013.

En este contexto, según el Ministerio de Energía, de los 17230 Gwh que se generaron en 2013 en el SING, 14101 se hicieron con base en carbón, representando $81.84 \%$ del total. En el caso del Sistema Interconectado Central, de los 50820 Gwh generados durante el año 2013, 11837 se hicieron con base en carbón. Sin embargo, su participación en la generación fue de $23.29 \%$, la más importante de todas las fuentes primarias, superando incluso a la hidráulica de embalse (18.78\%), el gas natural (18.99\%) y la hidráulica de pasada (19.48\%). Todo esto pese a que la capacidad instalada hasta diciembre de 2013 fuese mayoritariamente de hidráulica de embalse.

Según la Comisión Nacional de Energía (2016a), el incremento significativo del consumo de carbón se inició en 2010 con la recién inaugurada Central Nueva Ventanas, de la empresa Eléctrica Ventanas, en octubre de 2009. El consumo promedio de esta central supera cada mes 60000 toneladas de carbón. Luego de esta, en septiembre de 2011 se inauguró la central Santa María, de la empresa Compañía de Generación IndustrialIndustria Azucarera Nacional, S. A., que consume mensualmente más de 70000 toneladas. En diciembre de 2012 se inauguró la central Campiche, de la empresa AES Gener, que consume mensualmente un promedio superior a 60000 toneladas. Finalmente, en abril de 2012 entró en funcionamiento la central Bocamina 2, de la compañía Guacolda. Durante el resto de ese año, la central sólo consumió 166000 toneladas, mientras que, en 2013, su consumo total fue de 706000 toneladas. Igual situación ocurrió en el SING, con la creación de la central termoeléctrica Angamos, de la empresa Angamos que, desde 2013, ha contribuido con un consumo promedio mensual de 119000 toneladas de carbón. La construcción de estas megacentrales termoeléctricas, junto a las crisis de abastecimiento energético chileno, provocaron el periodo de mayor consumo de carbón en la historia de la energía en Chile.

$\mathrm{El}$ análisis de los bruscos cambios acontecidos en el consumo de energías primarias aplicadas a la generación de electricidad nos sugiere dos características de la economía chilena que merecen ser explicadas: en primer lugar, la dificultad para sostener el consumo estable de las energías prima- 
rias y, en segundo lugar, la capacidad del sector generador para adaptarse rápidamente a los cambios en la oferta de energía primaria. Ambas situaciones muestran discrepancias en cuanto a las funciones tanto del Estado como del sector privado. Para el primer caso, un estudio más acabado del impacto de las políticas públicas podría ayudar a comprender mejor esta situación; mientras que, para el segundo, un análisis más técnico del funcionamiento de los transformadores energéticos sería el indicado para entender esta característica.

Como se ha señalado anteriormente, durante los últimos diez años aproximadamente, el consumo de energías primarias en los centros de transformación se ha caracterizado por una volatilidad muy elevada. Esta falta de constancia en el consumo de un energético y la sustitución casi inmediata (menos de dos años) de una energía por otra, se debe principalmente a las características de las centrales termoeléctricas construidas en Chile. Cada central térmica cuenta con más de una unidad generadora de electricidad. Estas unidades utilizan un tipo de energía para producir la electricidad. Sin embargo, las centrales pueden tener unidades que consuman carbón, gas, petróleo y también biomasa. De esta forma, y por citar un ejemplo, la Unidad Uno de la Central Térmica Mejillones de la empresa Energía Chile (E-CL) puede generar electricidad con base en carbón, carbón más petcoke y fuel oil número seis, intercalando dichos combustibles dependiendo de las realidades de cada energético. Esto ha permitido a las generadoras chilenas adaptarse rápidamente a los cambios en los precios o en la oferta de las energías primarias, como han sido los casos del gas, petróleo y carbón. Ahora bien, el intervalo entre distintos tipos de energéticos se realiza, en el caso del gas (tanto gas natural licuado o gas licuado de petróleo), sólo con petróleo diesel. Esta situación se produce mayoritariamente en el Sistema Interconectado Central (19 centrales) y menos en el SING (seis centrales). En el caso del carbón, su complemento se produce generalmente con petcoke y fuel oil número 6 , pero nunca con gas. En este sentido, la sustitución del gas o del petróleo sólo se produce entre ellos y nunca con carbón. Es decir, el carbón no reemplazaría la generación de electricidad en las mismas centrales, sino que habría algunas centrales que dejarían de generar producto de la falta de energía primaria, incrementando la producción aquellas centrales consumidoras de carbón.

\section{LA DEPENDENCIA ENERGÉTICA: LA ETAPA DEL CARBÓN IMPORTADO}

Uno de los fenómenos que caracteriza este tercer ciclo es la existencia de una alta dependencia de la importación de energías primarias como base de la matriz energética chilena. Este fenómeno afectó mayoritariamente a 
tres de las energías primarias (petróleo, gas y carbón mineral), producto de la escasa producción chilena de los energéticos señalados. Diferente fue el caso de la hidroelectricidad, la biomasa y otras energías renovables no convencionales que, por sus características actuales, resulta inconveniente importarlas.

Tal y como se señaló más arriba, desde 1990 se observa una notoria divergencia entre la producción y el consumo de carbón mineral, que duraría hasta la puesta en funcionamiento de la mina Invierno en 2012. Desde este año, la producción volvió a crecer, aliviando un poco la demanda por importaciones. Según los datos proporcionados por la Comisión Nacional de Energía, la producción de carbón mineral representó, en 1991, 72\% del consumo bruto. Hacia el año 2000, dicha cantidad creció a $-8 \%$, promediando 8.4\% entre este año y 2012. En 2013, la cantidad representada por la producción ascendería a $20 \%$ del total consumido.

Con una producción agotada, el incremento de la demanda se resolvería recurriendo a la importación de energías. Durante las dos décadas señaladas, las importaciones representaron un promedio cercano a $88 \%$ del consumo total de carbón. Su incremento respondió necesariamente a la creciente demanda de carbón del sector eléctrico, presionado por la modernización de los sectores residencial e industrial y por el incremento de la producción de cobre electrolítico, altamente intenso en electricidad. La importación de carbón, junto con las demás energías, fue posible en gran parte gracias al incremento de los ingresos derivados de las exportaciones, situación que habría permitido disponer de divisas suficientes para satisfacer las necesidades energéticas. ${ }^{18}$

La producción desarrollada durante los últimos años por la mina Invierno ha supuesto un alivio a las importaciones de carbón, aunque ha presentado un panorama negativo que plantea preocupación. Pese a que la mayor parte de la producción de la minera está destinada a la exportación, en 2013, 34\% de ella fue distribuida en el comercio chileno, correspondientes a 744000 toneladas. Tal cantidad incrementó a 1746360 toneladas en 2014, correspondientes a $44 \%$ de la producción de la empresa. Sin embargo, debido a la reducción constante que ha sufrido el precio del carbón en el mundo, la producción de la mina Invierno ha ido disminuyendo desde 2015. Según la empresa, ${ }^{19}$ para 2016 se pronosticó

${ }^{18}$ Según el Banco Central, los ingresos producto de las exportaciones de bienes se duplicaron entre 1991 y 1995, para luego estancarse hasta 2002. Entre 2002 y 2007, tales ingresos se cuadriplicaron, para luego entrar en una nueva fase de estancamiento y crecimiento relativo hasta 2013. Estadísticas, Banco Central [en línea]. Recuperado de http://si3.bcentral.cl/estadisticas/Principal1/informes/SE/BDP/Anuarios.html [Consulta: 19 de octubre de 2016.]

${ }^{19}$ Recuperado de http://www.minainvierno.cl/mina-invierno-ajusta-dotacion-ante-menorproduccion-de-carbon-2/ 
una producción de 2300000 toneladas de carbón, esto es 25\% menos que la producción de 2015 .

Finalmente, una alternativa interesante a la dependencia energética de las energías primarias es la que plantea la generación eléctrica de las energías renovables no convencionales. En este sentido, una particularidad de estas energías es que su producción se encuentra destinada exclusivamente al consumo interno. Del mismo modo, las importaciones de estas energías son, en estos momentos, inexistentes. La importancia de estas energías en la matriz energética chilena ha aumentado de $28 \%$ del consumo total de energías primarias en 1991, a 34\% en 2013. La mayor parte de ellas corresponde a leña y biomasa, que incrementaron su participación en el consumo total de energías en Chile de 20 a $29 \%$ en el mismo periodo. Pese a este importante incremento, las señales siguen siendo aún muy débiles para adelantar pronósticos certeros.

\section{CONCLUSiOnes}

El estudio del tercer ciclo del carbón viene a completar una trayectoria de investigaciones que han estudiado la historia energética chilena desde 1844. En esta línea, el tercer ciclo del carbón sería un periodo completamente diferente a lo que la historia nos había mostrado en materia energética. Las principales características evidenciadas durante el segundo ciclo cambian radicalmente durante esta tercera etapa, situación que daría cuenta de una nueva realidad de la estructura energética nacional, caracterizada por un incremento importante del consumo de carbón mineral por la economía chilena, que pasa de 1000 a casi 8000 KTEP en los últimos 30 años, marcando la cifra más alta de la historia, un aumento importante de la dependencia energética y una creciente especialización de los usos del combustible para producir electricidad.

El empuje que adquirió el consumo de carbón fósil a partir de la década de 1980 coincide con el agotamiento de las minas chilenas localizadas en las regiones de Concepción y Arauco, por lo que las nuevas demandas de combustibles se tuvieron que satisfacer mediante importaciones, aumentando la dependencia energética del país. La explotación de la mina Invierno, localizada en la región austral de Magallanes, ha comenzado a revertir esta situación parcialmente.

En este tercer ciclo, el carbón tiende a concentrarse casi exclusivamente en la generación de electricidad, mientras el consumo final de carbón se redujo hasta casi desaparecer. Esta especialización del carbón en la generación eléctrica formaría parte de una nueva tendencia mundial en el uso de las energías, caracterizada por el incremento de la eficiencia energética. 
La sustitución del consumo final de carbón por la energía eléctrica forma parte de un importante proceso modernizador que habría afectado no sólo a los principales sectores productivos de la economía nacional, sino que también al sector comercial, público y residencial, gracias a un importante y profundo fenómeno urbanizador. La urbanización chilena impactó fuertemente en la demanda de electricidad, situación que coincidió con las crisis del petróleo, que limitaron fuertemente el abastecimiento del combustible. La solución a este problema se encontró en la generación hidroeléctrica, que sería estimulada desde la principal empresa estatal generadora de electricidad, ENDESA. Esta apuesta por diversificar la matriz eléctrica se vio fuertemente afectada por sucesivas crisis climáticas y conflictos externos, que limitaron las opciones energéticas de la economía chilena. Para continuar con la generación eléctrica, las empresas generadoras recurrieron al carbón mineral de importación, explicando los notables incrementos en el consumo de carbón que caracterizan este tercer ciclo.

\section{LISTA DE REFERENCIAS}

BadíA-Miró, M. y YÁÑEz, C. (2015). Localisation of industry in Chile, 1895-1967: Mining cycles and state policy. Australian Economic History Review, 55(3), 256-276. DOI: 10.1111/aehr.12074

Banco Central de Chile (2001). Indicadores económicos y sociales de Chile 1960-2000. Santiago: Departamento Publicaciones de la Gerencia de Investigación Económica. Bértola, L. (2011). Bolivia (Estado Plurinacional de), Chile y Perú desde la independencia: una historia de conflictos, transformaciones, inercias y desigualdad. En L. BÉRTOLA y P. GERCHUNOFF, Institucionalidad y desarrollo económico en América Latina (pp. 227-286). Santiago: Comisión Económica para América Latina y el Caribe.

Bértola, L. y OcAmpo, J. A. (2010). Desarrollo, vaivenes y desigualdad: una historia económica de América Latina desde la independencia. Madrid: Secretaría General Iberoamericana.

Bertoni, R. (2010). Energía y desarrollo: la restricción energética en Uruguay como problema (1882-2000) (Tesis doctoral). Universidad de la República, Uruguay.

Bertoni, R. y Román, C. (2013). Auge y ocaso del carbón mineral en Uruguay. Un análisis histórico desde fines del siglo XIX hasta la actualidad. Revista de Historia Económica-Journal of Latin American Economic History, 31(3), 459-497. DOI: 10.1017/ S0212610913000207

Bertoni, R. y Willebald, H. (2015). Do natural energy endowments matter? New Zealand and Uruguay in a comparative perspective, 1870-1940. Australian Economic History Reviewe, 56(1), 70-99. DOI: 10.1111/aehr.12092

Dirección General de Estadística (diversos años). Anuario Estadístico de la República de Chile. Santiago de Chile. 
Corporación de Fomento de la Producción (Corfo), Chile (1939). Fomento de la producción de energía eléctrica. Santiago: Nacimiento.

Comisión Nacional de Energía (1980). Balance de energía, 1960-1978. Santiago: Autor.

Comisión Nacional de Energía (1987). Balance energético, 1967-1986. Santiago: Autor. Comisión Nacional de Energía (1993). Balance energético, 1973-1992. Santiago: Autor.

Comisión Nacional de Energía (2016a). Consumo de energía primaria en centros de generación eléctrica del Sistema Interconectado Central (SIC) y Sistema Interconectado del Norte Grande (SING). Recuperado de http://www.cne.cl/estadisticas/electricidad/ [Consulta: 26 de octubre de 2016.]

Comisión Nacional de Energía (2016b). Balances nacionales de energía. Recuperado de la página de Internet de la Comisión Nacional de Energía: http://energiaabierta.cl/balance-energetico/ [Consulta: 26 de octubre de 2016.]

Empresa Nacional de Electricidad, S. A. (1993). ENDESA: 50 años. Santiago: Lord Cochrane.

Folchi, M. y Rubio, M. d. M. (2006). El consumo de energía fósil y la especificidad de la transición energética en América Latina, 1900-1930. III Simposio Latinoamericano y Caribeño de Historia Ambiental. Carmona, Sevilla.

Freeze, B. (2003). Coal. A human history. Londres: Penguin Books.

GARrido, M. (2015). El consumo de carbón en Chile. 1933-1960 (Tesis de magíster). Universidad de Valparaíso, Chile.

Instituto de Ingenieros de Chile (1988). Política eléctrica. Santiago: Editorial Universitaria.

JOFRÉ, J. (2012). Patrones de consumo aparente de energías modernas en América Latina, 18902003 (Tesis doctoral). Universitat de Barcelona, España.

Jones, D. W. (1991). How urbanization affects energy-use in developing countries. Energy Policy, 19(7), 621-630. DOI: 10.1016/0301-4215(91)90094-5

Kalder, A., Malanima, P. y Warde, P. (2014). Power to the people: Energy in Europe over the last five centuries. Princeton: Princeton University Press.

Meller, P. (1996). Un siglo de economía politica en Chile (1890-1990). Santiago: Andrés Bello.

MuÑoz, O. (1995). Los inesperados caminos de la modernización económica. Santiago: Universidad de Santiago.

PhilipPi, B. (1980). Síntesis de la situación energética de Chile. En H. MuÑOz (ed.), Desarrollo energético en América Latina y la economía mundial (pp. 90-113). Santiago: Editorial Universitaria.

Rubio, M. d. M. y Bertoni, R. (eds.) (2008). Energía y desarrollo en el largo siglo XX. Uruguay en el marco latinoamericano. Montevideo: Universitat Pompeu Fabra/Universidad de la República.

Rubio, M. d. M., Yáñez, C., Folchi, M., y Carreras, A. (2009). Energy as an indicator of modernization in Latin America, 1890-1925. The Economic History Review, 63(3), 768-804. DOI: 10.1111/j.1468-0289.2009.00463.x 
Rubio, M. d. M. y Folchi, M. (2012). Will small energy consumers be faster in transition? Evidence from the early shift from coal to oil in Latin America. Energy Policy, 50, 50-61. doi:10.1016/j.enpol.2012.03.054

Rubio, M. d. M. y TAfunell, X. (2014). Latin American hydropower: A century of uneven evolution. Renewable and Sustainable Energy Reviews, 38, 323-334. DOI: 10.1016/J.RSER.2014.05.068

SmiL, V. (2010). Energy transitions: History, requirements, prospects. Santa Barbara: Praeger Publishers.

Stevens, P. (2000). The economics of energy. Reino Unido: Edward Elgar Publishing.

Walpole, S. C., Prieto-Merino, D., Edwards, Ph., Cleland, J., Stevens, G. y RoBERTS, I. (2012). The weight of nations: An estimation of adult human biomass. BioMed Central Public Health, 12(439), 1-6. DOI: 10.1186/1471-2458-12-439

Wrigley, E. P. (2013). Energy and the English industrial revolution. Londres: Cambridge University Press.

YÁÑEZ, C. (2012). Economic modernization in adverse institutional environments: The case of Cuba and Chile. En C. YÁñEz y A. CARreras, The economies of Latin America. New cliometric data (pp. 105-118). Londres: Pickering \& Chatto.

YÁÑEz, C. y CARRERAS, A. (2012). Introduction: Latin American backwardness revisited. En C. Yáñez y A. Carreras, The economies of Latin America. New cliometric data (pp. 7-18). Londres: Pickering \& Chatto.

YÁÑEz, C. y Garrido, M. (2015). El consumo de carbón en Chile: 1933-1960. Transición energética y cambio estructural. Revista Uruguaya de Historia Económica, 5(8), 76-95.

YÁÑEz, C. y JofRÉ, J. (2011). Modernización económica y consumo energético en Chile, 1844-1930. Historia 396, 1(1), 127-156. Recuperado de http://www.historia396. cl/index.php/historia396/article/view/4/3

Yañez, C., Rubio, M. d. M., Jofré, J. y Carreras, A. (2013). El consumo de carbón mineral en América Latina. 1841-2000. Una historia de progreso y frustración. Revista de Historia Industrial, 53, 25-77. Recuperado de http://www.raco.cat/index. php/HistoriaIndustrial/article/view/271335 